\title{
NEAREST-NEIGHBOR GRAPHS ON THE CANTOR SET
}

\author{
NATHAN SHANK, ${ }^{*}$ Moravian College
}

\begin{abstract}
Let $\mathcal{X}_{n}$ be a collection of $n$ uniform, independent, and identically distributed points on the Cantor ternary set. We consider the asymptotics for the expected total edge length of the directed and undirected nearest-neighbor graph on $x_{n}$. We prove convergence to a constant of the rescaled expected total edge length of this random graph. The rescaling factor is a function of the fractal dimension and has a log-periodic, nonconstant behavior.

Keywords: Nearest neighbor; Cantor set; law of large numbers

2000 Mathematics Subject Classification: Primary 60F05
\end{abstract}

Secondary 60D05

\section{Introduction}

\subsection{Nearest-neighbor graphs on the Cantor set}

The purpose of this paper is to consider the asymptotics of the expected total edge length of the nearest-neighbor graph on vertices which are uniform, independent, and identically distributed (i.i.d.) on the Cantor ternary set, here denoted by $\mathcal{C}$. Recall that $\mathcal{C}$ is the set of points in $[0,1]$ whose ternary expansion does not contain the digit 1 .

There are two types of nearest-neighbor graph: the undirected nearest-neighbor graph and the directed nearest-neighbor graph. To construct these graphs, let $V_{n}:=\left\{v_{1}, v_{2}, \ldots, v_{n}\right\}$ be a set of $n$ vertices in $\mathbb{R}^{d}$. The nearest neighbor of $v_{i}$ is the vertex $v_{j} \in V_{n}-\left\{v_{i}\right\}$ such that $\left|v_{i}-v_{j}\right|=\min _{1 \leq k \leq n, k \neq i}\left|v_{i}-v_{k}\right|$. To ensure that the nearest neighbor of $v_{i}$ is unique, if there are ties, choose the nearest neighbor of $v_{i}$ to be the vertex with the largest index. Let $e\left(v_{i}\right)$ be the directed edge from $v_{i}$ to its nearest neighbor. The directed nearest-neighbor graph on $V$, denoted by $N^{\mathrm{D}}(V)$, is the graph $\langle V, E>$ where $E:=\{e(v) \mid v \in V\}$. Consideration of the undirected graph of $N^{\mathrm{D}}(V)$ produces the undirected nearest-neighbor graph on $V$, here denoted by $N(V)$. Throughout this paper, we will consider the undirected nearest-neighbor graph on uniform i.i.d. points in $\mathcal{C}$; however, all the results easily extend to the directed nearest-neighbor graph on such point sets.

Much attention has been given to showing asymptotics for the total edge length of graphs whose vertices are distributed on cubes or other well-behaved convex subsets of $\mathbb{R}^{d}$ (see, e.g. [2], [10], [11], [12], [14, pp. 97-109], and [15, pp. 27-51]).

Penrose and Yukich [11], [12] introduced the idea of stabilization and used this concept to prove convergence of expectations, laws of large numbers, and central limit theorems for stabilizing functionals. A functional defined on a graph is stabilizing if the local behavior of the graph at a vertex, $v$, is unaffected by changes to vertices outside a random, finite radius ball centered at $v$.

Received 28 November 2007; revision received 16 January 2009.

* Postal address: Mathematics and Computer Science Department, 1200 Main Street, Bethlehem, PA 18018, USA.

Email address: shank@math.moravian.edu 
Using stabilization, Penrose and Yukich [11, Theorem 3.2] proved a general law of large numbers for the total edge length of the $k$-nearest-neighbor graph for points which are uniformly distributed on cubes and other well-behaved subsets of $\mathbb{R}^{d}$. Let $\operatorname{Po}(n)$ be a Poisson random variable with mean $n$. Let the total edge length of the nearest-neighbor graph on a collection of $\operatorname{Po}(n)$ points which are uniformly distributed on $[0,1]^{d}$ be denoted by $\mathcal{T}(\operatorname{Po}(n))$. A corollary of Theorem 3.2 of [11] and Theorem 2.1 of [12] is

$$
\lim _{n \rightarrow \infty} n^{1 / d-1} \mathrm{E}[\mathcal{T}(\operatorname{Po}(n))]=\mathrm{E}\left[d\left(0, \mathcal{P}_{1}\right)\right],
$$

where $d\left(x, \mathcal{P}_{1}\right)$ is the distance from the point $x$ to its nearest neighbor in a Poisson point process of intensity 1 on $\mathbb{R}^{d}$. More generally, Theorem 2.1 of [12] proves a similar law of large numbers for nonuniform points.

We cannot simply apply Theorem 3.2 of [11] because the theorem requires the points to be uniformly distributed over a sequence of subsets of $\mathbb{R}^{d}$ with particular conditions on the size, boundary, limit, and diameter. Fractal subsets of $\mathbb{R}^{d}$ do not satisfy these conditions. We could consider the uniform distribution over a bounded fractal subset of $\mathbb{R}^{d}$ to be a special nonuniform distribution over a bounded convex subset of $\mathbb{R}^{d}$, and then apply Theorem 2.1 of [12]. However, Theorem 2.1 of [12] requires the points to be i.i.d. with common density function $f$. The uniform distribution over a fractal, such as the Cantor set, does not have a density function and, in fact, is a singular distribution (see Example 1.7 of [5]). Thus, [11] and [12] do not directly apply to uniform points on fractals.

One still might expect the proofs to extend to the case where the vertices are distributed uniformly on fractal subsets of $\mathbb{R}^{d}$. A close examination of graphs distributed on cubes exposes two difficulties that arise in generalizing the proofs of [11] and [12] to the fractal setting. One of the key features is scaling from a graph whose vertices are distributed with intensity $n$ per unit square to a graph whose vertices are distributed with intensity $n$ on a square of area $n$. The scaling will not work the same way in $\mathcal{C}$. In order to do so, the scaled Cantor set would have to contain length 1 Cantor sets, just as a scaled cube contains unit cubes. This happens only when $\mathcal{C}$ is scaled by a power of 3 . Scaling by a power of 3 may not reflect a Poisson point process of intensity 1 on a length 1 Cantor set. Another reason the same approach will not work in the fractal setting pertains to finding a typical vertex in an infinite Cantor set. The origin is a typical vertex in $\mathbb{R}^{d}$, whereas it is unclear what constitutes a typical vertex in the infinite Cantor set.

\subsection{Asymptotics involving log-periodic scaling}

Asymptotics for functionals on i.i.d. point sets usually involves scaling by volume or surface order terms. There have been a few results which seem to require scaling by log-periodic functions, although rigorously showing that the log-periodic functions are not constant has proved challenging. For example, Platzman and Bartholdi [13] considered a space-filling curve heuristic for the traveling salesman problem for vertices which are uniformly distributed in $[0,1]^{2}$. They designed a specific space-filling curve $\psi:[0,1] \rightarrow[0,1]^{2}$, then considered $n$ uniform i.i.d. vertices $v_{1}, v_{2}, \ldots, v_{n} \in[0,1]^{2}$. The space-filling curve heuristic involves constructing a tour through $n$ vertices, $V_{n}:=\left\{v_{1}, v_{2}, \ldots, v_{n}\right\}$, which visits the vertices in the order of their pre-image under $\psi$ in $[0,1]$. This is often called the space-filling curve tour. Let $L_{\psi}^{\mathrm{SFC}}(n)$ denote the total edge length of this tour on $n$ vertices which are uniformly distributed in $[0,1]^{2}$. In $[13]$ the authors argued that

$$
\liminf _{n \rightarrow \infty} n^{-1 / 2} \mathrm{E}\left[L_{\psi}^{\mathrm{SFC}}(n)\right]<\limsup _{n \rightarrow \infty} n^{-1 / 2} \mathrm{E}\left[L_{\psi}^{\mathrm{SFC}}(n)\right] .
$$




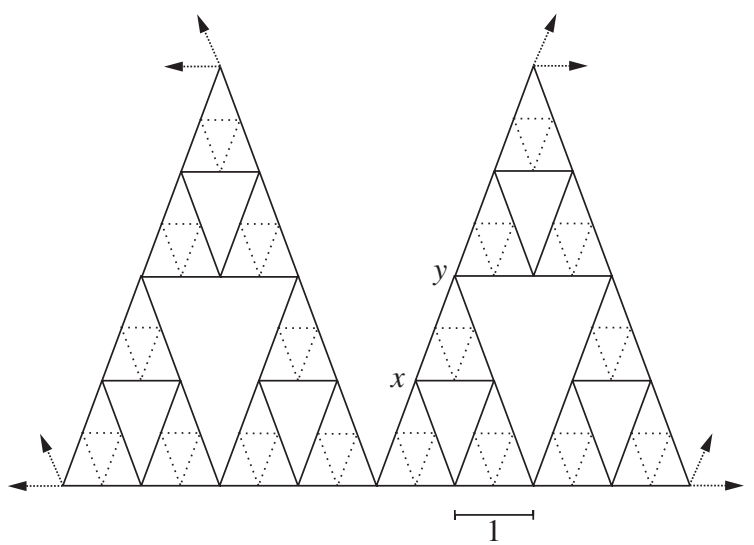

Figure 1: An infinite Sierpinski triangle.

In 1994, Gao and Steele [6] generalized the result of Platzman and Bartholdi to a class of space-filling curves with fractal-like properties. They showed that, under certain conditions on the space-filling curve $\psi$, there exists a periodic function $\phi_{\psi}: \mathbb{R}^{+} \rightarrow \mathbb{R}$ and a $p:=p(\psi)>0$ such that

$$
\lim _{n \rightarrow \infty} n^{-1 / 2} \phi_{\psi}\left(\log _{p}(n)\right) \mathrm{E}\left[L_{\psi}^{\mathrm{SFC}}(n)\right]=1 .
$$

Gao and Steele did not address the issue of nonconstant $\phi$, i.e. their methods admit the possibility that $\phi$ is identically constant.

In 1990, Lalley [9] considered (in much more generality) the total edge length of the traveling salesman tour on $n$ vertices (denoted by $L^{\mathrm{TSP}}(n)$ ) which are uniform i.i.d. on the Sierpinski triangle. He showed that there exists a continuous $\log _{3}$-periodic, positive function $\phi$ such that

$$
\lim _{n \rightarrow \infty} n^{\ln 2 / \ln 3-1} \phi(n) L^{\mathrm{TSP}}(n)=1 \quad \text { almost surely. }
$$

In this paper we consider the convergence of expectations, whereas Lalley's result is actually a law of large numbers. However, Lalley does not rule out the possibility of constant $\phi$.

In 1997, Gabner and Woess [7] considered the $n$-step transition probabilities on an infinite Sierpinski triangle; see Figure 1.

Let $p(x, y)=\frac{1}{4}$ if $|x-y|=1$. Let $X_{i}$ be the location at time $i$. Define the transition probabilities by $p^{(n)}(a, b)=\mathrm{P}\left(X_{n}=b \mid X_{0}=a\right)$. Gabner and Woess showed that $\lim _{n \rightarrow \infty} n^{\ln 3 / \ln 5} p^{(n)}(0,0)$ is periodic and nonconstant.

In 1996, Knopfmacher and Prodinger [8] used Rice's method to develop asymptotics for the expected value of the minimum order statistic on $\mathcal{C}$. They considered $M(n):=\min _{v_{i} \in V_{n}}\left|v_{i}\right|$, where $v_{i}$ are uniform i.i.d. on $\mathcal{C}$. Knopfmacher and Prodinger showed that there exists a $\log _{2}$-periodic, nonconstant function $\phi$ such that

$$
\lim _{n \rightarrow \infty} n^{1 / D} \mathrm{E}[M(\operatorname{Po}(n))] \phi(n)=1,
$$

where $D:=\ln 2 / \ln 3$ is the fractal dimension of $\mathcal{C}$.

Knopfmacher and Prodinger's method involves rewriting a recurrence relation as a sum of Bernoulli numbers. The summand is then expressed as a residue of a function which can be extended to the entire complex plane. Using the residue theorem, they showed that the sum of all the residues is 0 . The part of the summand that is not part of the $\mathrm{E}[M(\operatorname{Po}(n))]$ can easily be 
seen to be nonconstant. The argument is very creative and beautiful; however, it has not proved fruitful in the current problem.

Using a Tauberian theorem, we can extend the result of Knopfmacher and Prodinger to an infinite Sierpinski triangle. In every Sierpinski triangle of side length 1 , uniformly distribute an independent Poisson number of vertices with mean $n$. Denote the distance from the origin to its nearest neighbor by $M_{\triangle}(\operatorname{Po}(n))$. We can easily show that

$$
\lim _{n \rightarrow \infty} n^{\ln 2 / \ln 3} \mathrm{E}\left[M_{\triangle}(\operatorname{Po}(n))\right]
$$

is periodic and nonconstant.

The last two results suggest that the scaled expected total edge length of the nearest-neighbor graph on $n$ uniform i.i.d. points in $\mathcal{C}$ may have a nonconstant limit. On a unit cube, scaling by a factor of $n^{1 / d}$ produces a graph which is closely approximated by the infinite nearest-neighbor graph with intensity 1 per unit cube. In this setting, the average edge length of the scaled nearest-neighbor graph has the same asymptotics as the distance from the origin to its nearest neighbor in the infinite nearest-neighbor graph. If this intuition holds in the fractal setting then the average edge length of a scaled nearest-neighbor graph on the Cantor set would have the same asymptotics as the distance from the origin to its nearest neighbor in the graph defined on an infinite-size Cantor set with intensity 1 per unit Cantor set. Despite the previous examples which give intuition into the periodicity of the limit, the methods used to show a nonconstant limit do not carry over into the current problem. A new method will be used to show nonconstant periodicity of the limit.

\section{Main results}

\subsection{Definitions and setup}

Recall that $x \in \mathcal{C}$ if and only if $x \in[0,1]$ and $x=\sum_{i=1}^{\infty} a_{i} / 3^{i}$, where $a_{i} \in\{0,2\}$ for all $i$. Let $\mathcal{C}_{1}:=\mathcal{C} \cap\left[0, \frac{1}{3}\right]$ and $\mathcal{C}_{2}:=\mathcal{C} \cap\left[\frac{2}{3}, 1\right]$. For any bounded subset $S \subset \mathbb{R}$ admitting a uniform measure and any $k \in \mathbb{N}$, let $G(k, S):=G\left(\mathcal{X}_{k}, S\right)$ denote the undirected nearest-neighbor graph on $\mathcal{X}_{k}:=\left\{X_{1}, X_{2} \ldots X_{k}\right\}$, where the $X_{i}$ are uniform i.i.d. on $S$.

Let $L(k, S):=L(G(k, S))$ be the random variable representing the total edge length of the graph $G(k, S)$, and let $M(k, S)$ be the random variable denoting the distance between the origin and its nearest neighbor in $X_{k}$ if one exists. Throughout this paper, we adopt the convention that if $S$ is not specified then the underlying subset is $\mathcal{C}$. For example, $M(k):=M(k, \mathcal{C})$ and $L(k):=L(k, \mathcal{C})$.

\subsection{Main results}

The following theorem shows the nonconstant periodicity of the scaled expected total edge length of the nearest-neighbor graph on $\mathcal{C}$ with Poisson input.

Theorem 1. There is a $\log _{2}$-periodic, continuous, nonconstant function $\mathcal{L}: \mathbb{R}^{+} \rightarrow \mathbb{R}^{+}$such that

$$
\lim _{n \rightarrow \infty} n^{1 / D-1} \mathcal{L}(n) \mathrm{E}[L(\operatorname{Po}(n))]=1,
$$

where $D=\ln 2 / \ln 3$ is the fractal dimension of $\mathcal{C}$.

This result shows that there is a $\log _{2}$-periodic oscillation in the limit which is not present if the points are distributed on cubes or other well-behaved convex subsets of $\mathbb{R}^{d}$. In both settings, the average edge length scales like $n^{1-1 / d}$, where $d$ denotes the dimension. However, in the 
fractal setting, the average edge length behaves asymptotically like a log-periodic function times the expected distance from the 'origin' to its nearest neighbor. Not much is known about the function $\mathcal{L}$.

This leads to the following binomial version of Theorem 1 .

Theorem 2. We have

$$
\lim _{n \rightarrow \infty} n^{1 / D-1} \mathcal{L}(n) \mathrm{E}[L(n)]=1
$$

The proof of Theorem 1 relies heavily upon the recursive nature of $\mathcal{C}$. The self-similarity of $\mathcal{C}$ quickly leads to the existence of a limit. The difficulty arises in showing the nonconstant property of $\mathcal{L}$. The method used in the proof of the nonconstant property involves a recursion along with error terms. Note that $\mathrm{E}[L(n)]$ is approximately $2 / 3 \mathrm{E}[L(n / 2)]$ by scaling. To make this an equality, we have to introduce an error term, denoted by $f(n / 2)$. So, we have $\mathrm{E}[L(n)]=2 / 3 \mathrm{E}[L(n / 2)]+f(n / 2)$, where the error terms turn out to be computationally easy to handle. Repeating the recursion and evaluating the sum of the error terms allows us to analyze $\mathrm{E}[L(n)]$ and to establish the periodicity of $\mathcal{L}$.

The proof for the Poisson case in Theorem 1 will be provided in Section 3. Section 4 will produce an add-one cost which will be used in Section 5 to prove the binomial result in Theorem 2.

\section{Proof of Theorem 1}

\subsection{Outline of the proof}

The main idea of the proof is to develop a recursion for $\mathrm{E}[L(\operatorname{Po}(n))]$ in terms of $\mathrm{E}[L(\operatorname{Po}(n / 2))]$, and an error term. By repeating this recursion and collecting all the error terms, we successfully find the asymptotics for $\mathrm{E}[L(\operatorname{Po}(n))]$. The proof is completed in the following five steps. Steps 1 and 2 are used to prove the existence of the periodic function $\mathcal{L}$. Step 3 involves proving that the limit is nonconstant. In Step 4 we prove that $\mathcal{L}$ is a continuous function. Step 5 completes the proof of Theorem 1.

Step 1. Find a recursive formula for $\mathrm{E}[L(\operatorname{Po}(\alpha))]$, where $\alpha \in \mathbb{R}^{+}$.

Step 2. Show that there exists a $\log _{2}$-periodic function $\tilde{\mathcal{L}}: \mathbb{R}^{+} \rightarrow \mathbb{R}$ such that, for all $\alpha \in \mathbb{R}^{+}$,

$$
\lim _{n \rightarrow \infty}\left(\alpha 2^{n}\right)^{1 / D-1} \mathrm{E}\left[L\left(\operatorname{Po}\left(\alpha 2^{n}\right)\right)\right]=\tilde{\mathcal{L}}(\alpha) .
$$

Step 3. Show that $\tilde{\mathcal{L}}$ is not constant.

Step 4. Show that $\tilde{\mathscr{L}}$ is continuous on $\mathbb{R}^{+}$and strictly positive.

Step 5. Set $\mathcal{L}(\alpha):=(\tilde{\mathscr{L}}(\alpha))^{-1}$, and show that

$$
\lim _{n \rightarrow \infty} n^{1 / D-1} \mathcal{L}(n) \mathrm{E}[L(\operatorname{Po}(n))]=1 .
$$

\subsection{Proof of Step 1}

The recursion consists of showing that

$$
\mathrm{E}[L(\operatorname{Po}(\alpha))]=\frac{2}{3} \mathrm{E}\left[L\left(\operatorname{Po}\left(\frac{\alpha}{2}\right)\right)\right]+f\left(\frac{\alpha}{2}\right),
$$


where, for $x \geq 0$,

$$
f(x):=\frac{2}{3} x \mathrm{e}^{-x} \mathrm{E}[M(\operatorname{Po}(x))]+x \mathrm{e}^{-x}-x \mathrm{e}^{-2 x}-\frac{2}{3} x^{2} \mathrm{e}^{-2 x} .
$$

The main tool used for showing (1) is the self-similarity and scaling of $\mathcal{C}$ with respect to $\mathcal{C}_{1}$ and $\mathcal{C}_{2}$. If there is a Poisson number of points with mean $\alpha$ on $\mathcal{C}$ then, by self-similarity of $\mathcal{C}$, there will be a Poisson number of points with mean $\alpha / 2$ on $\mathcal{C}_{1}$, and similarly for $\mathcal{C}_{2}$. Also, $\mathcal{C}_{1}=1 / 3 \mathcal{C}$, and similarly for $\mathcal{C}_{2}$ (modulo a translation). These observations allow us to approximate $\mathrm{E}[L(\mathrm{Po}(\alpha))]$ by $2 / 3 \mathrm{E}[L(\operatorname{Po}(\alpha / 2))]$. To make this exact, we include an error term, $f(\alpha / 2)$, shown in (1).

To prove (1), assume without loss of generality that $L(0)=L(1)=0$. That is to say that the length of the nearest-neighbor graph is nonzero on two or more vertices. Assume that $M(0)=0$. This is to say that if there are no vertices then the origin does not have a nearest neighbor. For any set $A$, let $|A|$ denote the cardinality of $A$ and let $\mathcal{P}(n):=\mathcal{X}_{\mathrm{Po}(n)}=\left\{X_{1}, X_{2}, \ldots X_{\mathrm{Po}(n)}\right\}$, where the vertices $X_{i}$ are uniform i.i.d. on $\mathcal{C}$. Let $\mathcal{X}_{k}^{(1)}:=\left\{X_{1}^{1}, X_{2}^{1}, \ldots, X_{k}^{1}\right\}$, where the vertices $X_{i}^{1}$ are uniform i.i.d. on $\mathcal{C}_{1}$, and let $X_{k}^{(2)}:=\left\{X_{1}^{2}, X_{2}^{2}, \ldots, X_{k}^{2}\right\}$, where the vertices $X_{i}^{2}$ are uniform i.i.d. on $\mathcal{C}_{2}$. Let $\Lambda(k, j):=L\left(\mathcal{X}_{k}^{(1)} \cup \mathcal{X}_{j}^{(2)}\right)$. Define the event

$$
A_{\alpha}(i, j):=\left[\left|\mathcal{P}(\alpha) \cap \mathcal{C}_{1}\right|=i\right] \cap\left[\left|\mathcal{P}(\alpha) \cap \mathcal{C}_{2}\right|=j\right] .
$$

The recursion relies upon the following basic identity:

$$
\mathrm{E}[L(\mathrm{Po}(2 \alpha))]=\sum_{i=0}^{\infty} \sum_{j=0}^{\infty} \mathrm{E}[\Lambda(i, j)] \mathrm{P}\left(A_{2 \alpha}(i, j)\right) .
$$

The minimum distance between the points in $\mathcal{C}_{1}$ and $\mathcal{C}_{2}$ is at least $\frac{1}{3}$, and the diameter of $\mathcal{C}_{1}$ and $\mathcal{C}_{2}$ is at most $\frac{1}{3}$. Thus, if $\left|\mathcal{P}(n) \cap \mathcal{C}_{1}\right| \neq 1$ and $\left|\mathcal{P}(n) \cap \mathcal{C}_{2}\right| \neq 1$ then, with probability 1 , the length of the nearest-neighbor graph on $\mathcal{P}(n)$ would be the length of the nearest-neighbor graph on $\mathcal{P}(n) \cap \mathcal{C}_{1}$ plus the length of the nearest-neighbor graph on $\mathcal{P}(n) \cap \mathcal{C}_{2}$. If $\left|\mathcal{P}(n) \cap \mathcal{C}_{1}\right|=1$ then the nearest-neighbor graph consists of an edge from the isolated vertex $\mathcal{P}(n) \cap \mathcal{C}_{1}$ to the minimum vertex in $\mathcal{P}(n) \cap \mathcal{C}_{2}$ (similarly for $\left.\left|\mathcal{P}(n) \cap \mathcal{C}_{2}\right|=1\right)$. Thus,

$$
\Lambda(i, j)=L\left(i, \mathcal{C}_{1}\right)+L\left(j, \mathcal{C}_{2}\right)+\mathbf{1}_{\{i=1 \text { or } j=1\}} D_{2 \alpha}(i, j),
$$

where $D_{2 \alpha}(i, j)=\min \left\{|y-x|: x \in \mathcal{P}(2 \alpha) \cap \mathcal{C}_{1}, y \in \mathcal{P}(2 \alpha) \cap \mathcal{C}_{2}\right\}$, if it exists, where $\left|\mathcal{P}(2 \alpha) \cap \mathcal{C}_{1}\right|=i$ and $\left|\mathcal{P}(2 \alpha) \cap \mathcal{C}_{2}\right|=j$. By self-similarity of $\mathcal{C}$ we have

$$
\mathrm{E}\left[L\left(i, \mathcal{C}_{1}\right)\right]=\frac{1}{3} \mathrm{E}[L(i, \mathcal{C})]=\frac{1}{3} \mathrm{E}[L(i)] \quad \text { and } \quad \mathrm{E}\left[L\left(j, \mathcal{C}_{2}\right)\right]=\frac{1}{3} \mathrm{E}[L(j, \mathcal{C})]=\frac{1}{3} \mathrm{E}[L(j)] .
$$

Taking expectations of (4) and applying (5), we see that

$$
\mathrm{E}[\Lambda(i, j)]=\frac{1}{3} \mathrm{E}[L(i)]+\frac{1}{3} \mathrm{E}[L(j)]+\mathrm{E}\left[\mathbf{1}_{\{i=1 \text { or } j=1\}} D_{2 \alpha}(i, j)\right] .
$$

To determine the value of $\mathrm{E}\left[\mathbf{1}_{\{i=1 \text { or } j=1\}} D_{2 \alpha}(i, j)\right]$, consider the following three cases.

Case $(i): i=0$ and $j=1$ or $i=1$ and $j=0$. In this case, $\mathrm{E}\left[D_{2 \alpha}(0,1)\right]=\mathrm{E}[L(1)]=0$ by definition. Similarly, $\mathrm{E}\left[D_{2 \alpha}(1,0)\right]=0$.

Case (ii): $i=1$ and $j \geq 2$ or $i \geq 2$ and $j=1$. By symmetry, the same argument will work for either $i=1$ and $j \geq 2$ or $i \geq 2$ and $j=1$; so, assume without loss of generality that $i=1$ and $j \geq 2$. Set $x:=\mathcal{C}_{1} \cap \mathcal{P}(2 \alpha)$. 
To connect $x$ to its nearest neighbor first add an edge between $x$ and $\frac{1}{3}$. Then add an edge between $\frac{1}{3}$ and $\frac{2}{3}$, and then add an edge between $\frac{2}{3}$ and the minimum point in $\mathcal{C}_{2} \cap \mathcal{P}(2 \alpha)$ which contains exactly $j$ points. Thus,

$$
\begin{aligned}
\mathrm{E}\left[D_{2 \alpha}(1, j)\right] & :=\mathrm{E}\left[\min _{y \in \mathcal{P}(2 \alpha) \cap \mathcal{C}_{2}}|y-x||| \mathcal{C}_{2} \cap \mathcal{P}(2 \alpha) \mid=j\right] \\
& =\mathrm{E}\left[\frac{1}{3}-x\right]+\frac{1}{3}+\mathrm{E}\left[\min \left\{\mathcal{C}_{2} \cap \mathcal{P}(2 \alpha)\right\}-\frac{2}{3}|| \mathcal{C}_{2} \cap \mathcal{P}(2 \alpha) \mid=j\right] \\
& =\frac{1}{3}(\mathrm{E}[M(1)]+1+\mathrm{E}[M(j)]),
\end{aligned}
$$

where the last equality follows by the self-similarity of $\mathcal{C}$ and by the definition of $\mathrm{E}[M(n)]$. Using the fact that $\mathrm{E}[M(1)]=\frac{1}{2}$ (by symmetry), we have

$$
\mathrm{E}\left[D_{2 \alpha}(1, j)\right]=\frac{1}{2}+\frac{1}{3} \mathrm{E}[M(j)]
$$

Case (iii): $i=1$ and $j=1$. Set $x:=\mathcal{C}_{1} \cap \mathcal{P}(2 \alpha)$ and $y:=\mathcal{C}_{2} \cap \mathcal{P}(2 \alpha)$. The length of the edge from $x$ to $y$ would equal the length of the edge from $x$ to $\frac{1}{3}$ plus the length of the edge from $\frac{1}{3}$ to $\frac{2}{3}$ plus the length of the edge from $\frac{2}{3}$ to $y$. Thus,

$$
\begin{aligned}
\mathrm{E}\left[D_{2 \alpha}(1,1)\right] & =\mathrm{E}[|y-x|] \\
& =\mathrm{E}\left[\frac{1}{3}-x\right]+\frac{1}{3}+\mathrm{E}\left[y-\frac{2}{3}\right] \\
& =\frac{1}{3}(\mathrm{E}[M(1)]+1+\mathrm{E}[M(1)]) .
\end{aligned}
$$

Since $\mathrm{E}[M(1)]=\frac{1}{2}$, we have $\mathrm{E}\left[D_{2 \alpha}(1,1)\right]=\frac{2}{3}$.

Applying (6) to identity (3) gives

$$
\begin{aligned}
\mathrm{E}[L(\mathrm{Po}(2 \alpha))]= & \sum_{i=0}^{\infty} \sum_{j=0}^{\infty} \mathrm{E}[\Lambda(i, j)] \mathrm{P}\left(A_{2 \alpha}(i, j)\right) \\
= & \frac{1}{3} \sum_{i=0}^{\infty} \sum_{j=0}^{\infty} \mathrm{E}[L(i)] \mathrm{P}\left(A_{2 \alpha}(i, j)\right)+\frac{1}{3} \sum_{i=0}^{\infty} \sum_{j=0}^{\infty} \mathrm{E}[L(j)] \mathrm{P}\left(A_{2 \alpha}(i, j)\right) \\
& +\sum_{i=0}^{\infty} \sum_{j=0}^{\infty} \mathrm{E}\left[\mathbf{1}_{\{i=1 \text { or } j=1\}} D_{2 \alpha}(i, j)\right] \mathrm{P}\left(A_{2 \alpha}(i, j)\right)
\end{aligned}
$$

The two events $\left|\mathcal{C}_{1} \cap \mathcal{P}(2 \alpha)\right|=i$ and $\left|\mathcal{C}_{2} \cap \mathcal{P}(2 \alpha)\right|=j$ are independent since $\mathcal{C}_{1}$ and $\mathcal{C}_{2}$ are disjoint. Thus, if there is a mean of $2 \alpha$ vertices on $\mathcal{C}$ then there will be a mean of $\alpha$ vertices on $\mathcal{C}_{1}$ and $\mathcal{C}_{2}$. Hence,

$$
\mathrm{P}\left[A_{2 \alpha}(i, j)\right]=\frac{\alpha^{i} \mathrm{e}^{-\alpha}}{i !} \frac{\alpha^{j} \mathrm{e}^{-\alpha}}{j !}=\frac{\alpha^{i+j} \mathrm{e}^{-2 \alpha}}{i ! j !} .
$$

Applying (8) to (7) shows that

$$
\begin{aligned}
\mathrm{E}[L(\mathrm{Po}(2 \alpha))]= & \frac{1}{3} \sum_{i=0}^{\infty} \sum_{j=0}^{\infty} \mathrm{E}[L(i)] \frac{\alpha^{i} \mathrm{e}^{-\alpha}}{i !} \frac{\alpha^{j} \mathrm{e}^{-\alpha}}{j !}+\frac{1}{3} \sum_{i=0}^{\infty} \sum_{j=0}^{\infty} \mathrm{E}[L(j)] \frac{\alpha^{i} \mathrm{e}^{-\alpha}}{i !} \frac{\alpha^{j} \mathrm{e}^{-\alpha}}{j !} \\
& +\sum_{i=0}^{\infty} \sum_{j=0}^{\infty} \mathrm{E}\left[\mathbf{1}_{\{i=1 \text { or } j=1\}} D_{2 \alpha}(i, j)\right] \frac{\alpha^{i} \mathrm{e}^{-\alpha}}{i !} \frac{\alpha^{j} \mathrm{e}^{-\alpha}}{j !} .
\end{aligned}
$$


By a change of variable, the first two double summations are equal. Also, by construction and by case (i) above, the nonzero terms of the last double summation occur only when $\min \{i, j\}=1$. Thus,

$$
\begin{aligned}
\mathrm{E}[L(\operatorname{Po}(2 \alpha))]= & \frac{2}{3} \sum_{i=0}^{\infty} \sum_{j=0}^{\infty} \mathrm{E}[L(j)] \frac{\alpha^{i} \mathrm{e}^{-\alpha}}{i !} \frac{\alpha^{j} \mathrm{e}^{-\alpha}}{j !}+\sum_{j=2}^{\infty} \mathrm{E}\left[D_{2 \alpha}(1, j)\right] \alpha \mathrm{e}^{-\alpha} \frac{\alpha^{j} \mathrm{e}^{-\alpha}}{j !} \\
& +\sum_{i=2}^{\infty} \mathrm{E}\left[D_{2 \alpha}(i, 1)\right] \alpha \mathrm{e}^{-\alpha} \frac{\alpha^{i} \mathrm{e}^{-\alpha}}{i !}+\mathrm{E}\left[D_{2 \alpha}(1,1)\right] \alpha \mathrm{e}^{-\alpha} \alpha \mathrm{e}^{-\alpha}
\end{aligned}
$$

By symmetry of $\mathcal{C}$, the last two summations are equal. Thus,

$$
\begin{aligned}
\mathrm{E}[L(\mathrm{Po}(2 \alpha))]= & \frac{2}{3} \sum_{i=0}^{\infty} \sum_{j=0}^{\infty} \mathrm{E}[L(j)] \frac{\alpha^{i} \mathrm{e}^{-\alpha}}{i !} \frac{\alpha^{j} \mathrm{e}^{-\alpha}}{j !}+2 \sum_{j=2}^{\infty} \mathrm{E}\left[D_{2 \alpha}(1, j)\right] \alpha \mathrm{e}^{-\alpha} \frac{\alpha^{j} \mathrm{e}^{-\alpha}}{j !} \\
& +\mathrm{E}\left[D_{2 \alpha}(1,1)\right] \alpha^{2} \mathrm{e}^{-2 \alpha} .
\end{aligned}
$$

Applying case (ii) and case (iii) shows that

$$
\begin{aligned}
\mathrm{E}[L(\operatorname{Po}(2 \alpha))]= & \frac{2}{3} \sum_{j=0}^{\infty} \mathrm{E}[L(j)] \frac{\alpha^{j} \mathrm{e}^{-\alpha}}{j !}+2 \sum_{j=2}^{\infty}\left(\frac{1}{2}+\frac{1}{3} \mathrm{E}[M(j)]\right) \alpha \mathrm{e}^{-\alpha} \frac{\alpha^{j} \mathrm{e}^{-\alpha}}{j !} \\
& +\frac{2}{3} \alpha^{2} \mathrm{e}^{-2 \alpha} .
\end{aligned}
$$

By extending the second summation to start at 0 and since

$$
\sum_{j=0}^{\infty} \frac{\alpha^{j} \mathrm{e}^{-\alpha}}{j !} \mathrm{E}[L(j)]=\mathrm{E}[L(\operatorname{Po}(\alpha))],
$$

and similarly for $\mathrm{E}[M(j)]$, we see that

$$
\begin{aligned}
\mathrm{E}[L(\operatorname{Po}(2 \alpha))]= & \frac{2}{3} \mathrm{E}[L(\operatorname{Po}(\alpha))]+\frac{2}{3} \alpha \mathrm{e}^{-\alpha} \mathrm{E}[M(\operatorname{Po}(\alpha))]+2 \alpha \mathrm{e}^{-\alpha} \sum_{j=2}^{\infty} \frac{1}{2} \frac{\alpha^{j} \mathrm{e}^{-\alpha}}{j !} \\
& -\frac{2}{3} \alpha \mathrm{e}^{-2 \alpha} \mathrm{E}[M(1)]+\frac{2}{3} \alpha^{2} \mathrm{e}^{-2 \alpha} .
\end{aligned}
$$

Expanding, combining, canceling, and using the fact that $\mathrm{E}[M(1)]=\frac{1}{2}$, we see that (9) simplifies to

$$
\mathrm{E}[L(\operatorname{Po}(2 \alpha))]=\frac{2}{3} \mathrm{E}[L(\operatorname{Po}(\alpha))]+\frac{2}{3} \alpha \mathrm{e}^{-\alpha} \mathrm{E}[M(\operatorname{Po}(\alpha))]+\alpha \mathrm{e}^{-\alpha}-\alpha \mathrm{e}^{-2 \alpha}-\frac{2}{3} \alpha^{2} \mathrm{e}^{-2 \alpha} .
$$

Replacing $2 \alpha$ by $\alpha$ yields

$$
\begin{aligned}
\mathrm{E}[L(\operatorname{Po}(\alpha))]= & \frac{2}{3} \mathrm{E}\left[L\left(\operatorname{Po}\left(\frac{\alpha}{2}\right)\right)\right]+\frac{\alpha}{3} \mathrm{e}^{-\alpha / 2} \mathrm{E}\left[M\left(\operatorname{Po}\left(\frac{\alpha}{2}\right)\right)\right]+\frac{\alpha}{2} \mathrm{e}^{-\alpha / 2}-\frac{\alpha}{2} \mathrm{e}^{-\alpha} \\
& -\frac{2}{3}\left(\frac{\alpha}{2}\right)^{2} \mathrm{e}^{-\alpha} \\
= & \frac{2}{3} \mathrm{E}\left[L\left(\operatorname{Po}\left(\frac{\alpha}{2}\right)\right)\right]+f\left(\frac{\alpha}{2}\right)
\end{aligned}
$$

by the definition of $f$ (see (2)). 
This shows the desired recursion for Step 1. Before continuing to Step 2, we note the following properties about the error function $f$. First note that, since $M[\operatorname{Po}(x)] \leq 1$ and all the terms exponentially decay in $x$, there exists a $\beta<\infty$ such that, for all $x \geq 0,|f(x)| \leq \beta$.

Simple calculus shows that $g(x)=\mathrm{e}^{x}-1-2 x / 3>0$ for $x>0$. Since $f(x)=$ $\frac{2}{3} x \mathrm{e}^{-x} \mathrm{E}[M(\operatorname{Po}(x))]+x \mathrm{e}^{-2 x} g(x)$, we see that

$$
f(x)>0 \text { for all } x>0 .
$$

\subsection{Proof of Step 2}

Fix $\alpha \in \mathbb{R}^{+}$and iterate (1) $n$ times to obtain

$$
\begin{aligned}
\mathrm{E}\left[L\left(\operatorname{Po}\left(\alpha 2^{n}\right)\right)\right] & =\frac{2}{3} \mathrm{E}\left[L\left(\operatorname{Po}\left(\alpha 2^{n-1}\right)\right)\right]+f\left(\alpha 2^{n-1}\right) \\
& =\left(\frac{2}{3}\right)^{2} \mathrm{E}\left[L\left(\operatorname{Po}\left(\alpha 2^{n-2}\right)\right)\right]+\frac{2}{3} f\left(\alpha 2^{n-2}\right)+f\left(\alpha 2^{n-1}\right) \\
& =\cdots \\
& =\left(\frac{2}{3}\right)^{n} \mathrm{E}[L(\operatorname{Po}(\alpha))]+\sum_{k=0}^{n-1}\left(\frac{2}{3}\right)^{n-k-1} f\left(\alpha 2^{k}\right) .
\end{aligned}
$$

Note that $2^{1 / D}=2^{\ln 3 / \ln 2}=3$. Multiplying both sides of the above by $\left(\alpha 2^{n}\right)^{1 / D-1}$ gives

$$
\left(\alpha 2^{n}\right)^{1 / D-1} \mathrm{E}\left[L\left(\operatorname{Po}\left(\alpha 2^{n}\right)\right)\right]=\alpha^{1 / D-1} \mathrm{E}[L(\operatorname{Po}(\alpha))]+\alpha^{1 / D-1} \sum_{k=0}^{n-1}\left(\frac{3}{2}\right)^{k+1} f\left(\alpha 2^{k}\right) .
$$

By (2), $f$ decays exponentially fast and, thus, as $n \rightarrow \infty$, the sum in (11) converges. Setting

$$
\tilde{\mathscr{L}}(\alpha):=\alpha^{1 / D-1} \mathrm{E}[L(\operatorname{Po}(\alpha))]+\alpha^{1 / D-1} \sum_{k=0}^{\infty}\left(\frac{3}{2}\right)^{k+1} f\left(\alpha 2^{k}\right)
$$

and taking limits as $n \rightarrow \infty$ on both sides of (11) gives, for all $\alpha \in \mathbb{R}^{+}$,

$$
\tilde{\mathcal{L}}(\alpha)=\lim _{n \rightarrow \infty}\left(\alpha 2^{n}\right)^{1 / D-1} \mathrm{E}\left[L\left(\operatorname{Po}\left(\alpha 2^{n}\right)\right)\right] .
$$

Note that $\tilde{\mathscr{L}}(2 \alpha)=\tilde{\mathscr{L}}(\alpha)$, i.e. $\tilde{\mathcal{L}}$ is $\log _{2}$ periodic, completing Step 2 .

\subsection{Proof of Step 3}

Since $\tilde{\mathscr{L}}$ is $\log _{2}$ periodic (13), it is clear that, for all $m \in \mathbb{N}$ and $\alpha \in \mathbb{R}^{+}$,

$$
\tilde{\mathscr{L}}(\alpha)=\tilde{\mathscr{L}}\left(\alpha 2^{-1}\right)=\tilde{\mathscr{L}}\left(\alpha 2^{-2}\right)=\cdots=\tilde{\mathscr{L}}\left(\alpha 2^{-m}\right) .
$$

Thus,

$$
\tilde{\mathcal{L}}(\alpha)=\lim _{m \rightarrow \infty} \tilde{\mathcal{L}}\left(\alpha 2^{-m}\right),
$$

showing that $\tilde{\mathscr{L}} \equiv c$ if and only if $\tilde{\mathscr{L}}$ is right continuous at the origin. To prove that $\tilde{\mathscr{L}}$ is not identically constant, it is enough to show that $\lim _{m \rightarrow \infty} \tilde{\mathcal{L}}\left(2^{-m}\right) \neq \lim _{m \rightarrow \infty} \tilde{\mathcal{L}}\left(3\left(2^{-m}\right)\right)$. We first find a general expression for $\tilde{\mathcal{L}}\left(\alpha\left(2^{-m}\right)\right)$ as follows. By (12) and (14),

$$
\begin{aligned}
\tilde{\mathscr{L}}(\alpha) & =\lim _{m \rightarrow \infty} \tilde{\mathcal{L}}\left(\alpha 2^{-m}\right) \\
& =\lim _{m \rightarrow \infty}\left[\left(\alpha 2^{-m}\right)^{1 / D-1} \mathrm{E}\left[L\left(\operatorname{Po}\left(\alpha 2^{-m}\right)\right)\right]+\left(\alpha 2^{-m}\right)^{1 / D-1} \sum_{k=0}^{\infty}\left(\frac{3}{2}\right)^{k+1} f\left(\alpha 2^{-m} 2^{k}\right)\right] .
\end{aligned}
$$


Applying the identity $2^{1 / D}=3$ shows that

$$
\tilde{\mathcal{L}}(\alpha)=\lim _{m \rightarrow \infty}\left[\left(\alpha 2^{-m}\right)^{1 / D-1} \mathrm{E}\left[L\left(\operatorname{Po}\left(\alpha 2^{-m}\right)\right)\right]+\alpha^{1 / D-1}\left(\frac{3}{2}\right) \sum_{k=0}^{\infty}\left(\frac{3}{2}\right)^{k-m} f\left(\alpha 2^{k-m}\right)\right] .
$$

Re-indexing the summation shows that

$$
\tilde{\mathcal{L}}(\alpha)=\lim _{m \rightarrow \infty}\left[\left(\alpha 2^{-m}\right)^{1 / D-1} \mathrm{E}\left[L\left(\operatorname{Po}\left(\alpha 2^{-m}\right)\right)\right]+\alpha^{1 / D-1}\left(\frac{3}{2}\right) \sum_{k=-m}^{\infty}\left(\frac{3}{2}\right)^{k} f\left(\alpha 2^{k}\right)\right] .
$$

The limit of the first term vanishes since $L$ is bounded. Note that the infinite series

$$
\sum_{k=-\infty}^{\infty}\left(\frac{3}{2}\right)^{k} f\left(\alpha 2^{k}\right)
$$

converges because (12) exists and because of the inequality

$$
\sum_{k=-\infty}^{-1}\left(\frac{3}{2}\right)^{k} f\left(\alpha 2^{k}\right)=\sum_{k=1}^{\infty}\left(\frac{2}{3}\right)^{k} f\left(\alpha 2^{-k}\right) \leq \beta \sum_{k=1}^{\infty}\left(\frac{2}{3}\right)^{k}<\infty,
$$

where $\beta$ is defined before (10). Thus, distributing the limit gives

$$
\tilde{\mathcal{L}}(\alpha)=\lim _{m \rightarrow \infty} \tilde{\mathcal{L}}\left(\alpha 2^{-m}\right)=\frac{3}{2} \alpha^{1 / D-1} \sum_{k=-\infty}^{\infty}\left(\frac{3}{2}\right)^{k} f\left(\alpha 2^{k}\right) .
$$

By the definition of $f$ (see (2)),

$$
\begin{aligned}
\tilde{L}(\alpha)=\frac{3}{2} \alpha^{1 / D-1} \sum_{k=-\infty}^{\infty}\left(\frac{3}{2}\right)^{k} & {\left[\frac{2}{3} \alpha 2^{k} \mathrm{e}^{-\alpha 2^{k}} \mathrm{E}\left[M\left(\operatorname{Po}\left(\alpha 2^{k}\right)\right)\right]+\alpha 2^{k} \mathrm{e}^{-\alpha 2^{k}}\right.} \\
& \left.-\alpha 2^{k} \mathrm{e}^{-2 \alpha 2^{k}}-\frac{2}{3} \alpha^{2} 2^{2 k} \mathrm{e}^{-2 \alpha 2^{k}}\right] .
\end{aligned}
$$

Distributing the sum and simplifying gives

$$
\begin{aligned}
\tilde{L}(\alpha)= & \alpha^{1 / D} \sum_{k=-\infty}^{\infty} 3^{k} \mathrm{e}^{-\alpha 2^{k}} \mathrm{E}\left[M\left(\operatorname{Po}\left(\alpha 2^{k}\right)\right)\right]+\frac{3}{2} \alpha^{1 / D} \sum_{k=-\infty}^{\infty} 3^{k} \mathrm{e}^{-\alpha 2^{k}} \\
& -\frac{3}{2} \alpha^{1 / D} \sum_{k=-\infty}^{\infty} 3^{k} \mathrm{e}^{-\alpha 2^{k+1}}-\alpha^{1 / D+1} \sum_{k=-\infty}^{\infty} 6^{k} \mathrm{e}^{-\alpha 2^{k+1}} .
\end{aligned}
$$

Re-indexing the third and fourth summations gives

$$
\begin{aligned}
\tilde{\mathscr{L}}(\alpha)= & \alpha^{1 / D} \sum_{k=-\infty}^{\infty} 3^{k} \mathrm{e}^{-\alpha 2^{k}} \mathrm{E}\left[M\left(\operatorname{Po}\left(\alpha 2^{k}\right)\right)\right]+\frac{3}{2} \alpha^{1 / D} \sum_{k=-\infty}^{\infty} 3^{k} \mathrm{e}^{-\alpha 2^{k}} \\
& -\frac{1}{2} \alpha^{1 / D} \sum_{k=-\infty}^{\infty} 3^{k} \mathrm{e}^{-\alpha 2^{k}}-\frac{1}{6} \alpha^{1 / D+1} \sum_{k=-\infty}^{\infty} 6^{k} \mathrm{e}^{-\alpha 2^{k}} .
\end{aligned}
$$


Combining the second and third summations produces

$$
\begin{aligned}
\tilde{\mathscr{L}}(\alpha)= & \alpha^{1 / D} \sum_{k=-\infty}^{\infty} 3^{k} \mathrm{e}^{-\alpha 2^{k}} \mathrm{E}\left[M\left(\operatorname{Po}\left(\alpha 2^{k}\right)\right)\right]+\alpha^{1 / D} \sum_{k=-\infty}^{\infty} 3^{k} \mathrm{e}^{-\alpha 2^{k}} \\
& -\frac{1}{6} \alpha^{1 / D+1} \sum_{k=-\infty}^{\infty} 6^{k} \mathrm{e}^{-\alpha 2^{k}} \\
:= & A(\alpha)+B(\alpha)-C(\alpha) .
\end{aligned}
$$

To show that $\tilde{\mathcal{L}}(1) \neq \tilde{\mathcal{L}}(3)$, employ (16) with $\alpha=1$ and $\alpha=3$, and show that

$$
\tilde{\mathscr{L}}(1)=A(1)+B(1)-C(1)<A(3)+B(3)-C(3)=\tilde{\mathscr{L}}(3) .
$$

3.4.1. Analysis of $\tilde{\mathscr{L}}(1)$. The idea is to obtain an upper bound, $\tilde{\mathscr{L}}^{\mathrm{u}}(1)$, for $\tilde{\mathscr{L}}(1)$. This will be accomplished by setting $\alpha=1$ in (16) and finding upper bounds for $A(1)$ and $B(1)$, and combining these with a lower bound for $C(1)$.

An upper bound for $A(1)$. To bound $A(1)$, we will split it into three parts and bound each part. Two parts will be bound using the exponential decay while the other part will be bound by using a finite number of terms. Conditioning on the value of the random number $\operatorname{Po}\left(2^{k}\right)$ shows that

$$
A(1):=\sum_{k=-\infty}^{\infty} 3^{k} \mathrm{e}^{-2^{k}} \mathrm{E}\left[M\left(\operatorname{Po}\left(2^{k}\right)\right)\right]=\sum_{k=-\infty}^{\infty} \sum_{j=0}^{\infty} 3^{k} \mathrm{e}^{-2^{k}} \frac{\mathrm{e}^{-2^{k}} 2^{k j}}{j !} \mathrm{E}[M(j)] .
$$

Splitting the infinite sum into a finite sum and two infinite sums shows that

$$
\begin{aligned}
A(1)= & \sum_{|k| \leq 20} \sum_{j=0}^{20} 3^{k} \mathrm{e}^{-2^{k+1}} 2^{k j} \frac{1}{j !} \mathrm{E}[M(j)]+\sum_{|k| \geq 21} \sum_{j=0}^{\infty} 3^{k} \mathrm{e}^{-2^{k+1}} 2^{k j} \frac{1}{j !} \mathrm{E}[M(j)] \\
& +\sum_{|k| \leq 20} \sum_{j=21}^{\infty} 3^{k} \mathrm{e}^{-2^{k+1}} 2^{k j} \frac{1}{j !} \mathrm{E}[M(j)] \\
:= & A_{a}(1)+A_{b}(1)+A_{c}(1) .
\end{aligned}
$$

We first find upper bounds for $A_{b}(1)$ and $A_{c}(1)$. Since $\mathrm{E}[M(j)] \leq 1$ for all $j>0$,

$$
A_{b}(1) \leq \sum_{|k| \geq 21} \sum_{j=0}^{\infty} 3^{k} \mathrm{e}^{-2^{k+1}} 2^{k j} \frac{1}{j !}=\sum_{|k| \geq 21} 3^{k} \mathrm{e}^{-2^{k+1}} \mathrm{e}^{2^{k}}=\sum_{|k| \geq 21} 3^{k} \mathrm{e}^{-2^{k}} .
$$

Splitting the sum over the parts where $k$ is positive and negative gives

$$
A_{b}(1) \leq \sum_{k=21}^{\infty} 3^{k} \mathrm{e}^{-2^{k}}+\sum_{k=21}^{\infty}\left(\frac{1}{3}\right)^{k} \mathrm{e}^{-2^{-k}}
$$

Note that if $k \geq 21$ then $2^{k}>2 k$, which implies that $\mathrm{e}^{-2^{k}} \leq \mathrm{e}^{-2 k}$. Thus,

$$
A_{b}(1) \leq \sum_{k=21}^{\infty}\left(\frac{3}{\mathrm{e}^{2}}\right)^{k}+\sum_{k=21}^{\infty}\left(\frac{1}{3}\right)^{k} \leq 2\left(\frac{3}{\mathrm{e}^{2}}\right)^{21}+\frac{1}{2 \cdot 3^{20}}
$$


Bounding $A_{c}(1)$ requires a little more work. By definition (17) and $\mathrm{E}[M(j)] \leq 1$ for all $j>0$,

$$
A_{c}(1):=\sum_{|k| \leq 20} \sum_{j=21}^{\infty} 3^{k} \mathrm{e}^{-2^{k+1}} 2^{k j} \frac{1}{j !} \mathrm{E}[M(j)] \leq \sum_{|k| \leq 20} \sum_{j=21}^{\infty} 3^{k} \mathrm{e}^{-2^{k+1}} 2^{k j} \frac{1}{j !} .
$$

Splitting the sum over the parts where $k$ is positive and negative gives

$$
\begin{aligned}
A_{c}(1) & \leq \sum_{k=0}^{20} \sum_{j=21}^{\infty} 3^{k} \mathrm{e}^{-2^{k+1}} 2^{k j} \frac{1}{j !}+\sum_{k=1}^{20} \sum_{j=21}^{\infty}\left(\frac{1}{3}\right)^{k} \mathrm{e}^{-2^{-k+1}} 2^{-k j} \frac{1}{j !} \\
& \leq \sum_{k=0}^{20} \sum_{j=21}^{\infty} 3^{k} \mathrm{e}^{-2^{k+1}} 2^{k j} \frac{1}{j !}+\sum_{k=1}^{20} \sum_{j=21}^{\infty}\left(\frac{1}{3}\right)^{k} 2^{-k j} \frac{1}{j !} \\
& =\sum_{k=0}^{20} 3^{k} \mathrm{e}^{-2^{k+1}} \sum_{j=21}^{\infty} \frac{2^{k j}}{j !}+\sum_{k=1}^{20}\left(\frac{1}{3}\right)^{k} \sum_{j=21}^{\infty}\left(\frac{1}{2}\right)^{k j} \frac{1}{j !} .
\end{aligned}
$$

If $j \geq 21$ then

$$
\left(\frac{1}{2}\right)^{k j} \frac{1}{j !} \leq \frac{1}{21 !}\left(\frac{1}{2}\right)^{21 k}\left(\frac{1}{2}\right)^{k(j-21)}
$$

Thus,

$$
\begin{aligned}
A_{c}(1) & \leq \sum_{k=0}^{20} 3^{k} \mathrm{e}^{-2^{k+1}}\left(\mathrm{e}^{2^{k}}-\sum_{j=0}^{20} \frac{2^{k j}}{j !}\right)+\sum_{k=1}^{20}\left(\frac{1}{3}\right)^{k}\left(\frac{1}{2}\right)^{21 k} \frac{1}{21 !} \sum_{j=21}^{\infty}\left(\frac{1}{2}\right)^{k(j-21)} \\
& =\sum_{k=0}^{20} 3^{k} \mathrm{e}^{-2^{k+1}} \mathrm{e}^{2^{k}}-\sum_{k=0}^{20} 3^{k} \mathrm{e}^{-2^{k+1}} \sum_{j=0}^{20} \frac{2^{k j}}{j !}+\sum_{k=1}^{20}\left(\frac{1}{3}\right)^{k}\left(\frac{1}{2}\right)^{21 k} \frac{1}{21 !} \sum_{j=21}^{\infty}\left(\frac{1}{2}\right)^{k(j-21)} .
\end{aligned}
$$

Simplifying and extending the third summation from $k=1, \ldots, 20$ to $k=1, \ldots, \infty$ and re-indexing the last summation gives

$$
A_{c}(1) \leq \sum_{k=0}^{20} 3^{k} \mathrm{e}^{-2^{k}}-\sum_{k=0}^{20} 3^{k} \mathrm{e}^{-2^{k+1}} \sum_{j=0}^{20} \frac{2^{k j}}{j !}+\frac{1}{21 !} \sum_{k=1}^{\infty}\left(\frac{1}{2}\right)^{21 k}\left(\frac{1}{3}\right)^{k} \sum_{j=0}^{\infty}\left(\frac{1}{2}\right)^{k j}
$$

It is easily seen that the double sum is bounded by $(1 / 21 !)\left(\frac{1}{2}\right)^{21}$; thus,

$$
A_{c}(1) \leq \sum_{k=0}^{20} 3^{k} \mathrm{e}^{-2^{k}}-\sum_{k=0}^{20} 3^{k} \mathrm{e}^{-2^{k+1}} \sum_{j=0}^{20} \frac{2^{k j}}{j !}+\frac{1}{21 !}\left(\frac{1}{2}\right)^{21} .
$$

Consider $h(x)=3^{x} \mathrm{e}^{-2^{x}}$. Clearly, $h(x)$ is decreasing when $x \geq 1$; thus, $h(k)=3^{k} \mathrm{e}^{-2^{k}} \leq$ $3^{5} \mathrm{e}^{-2^{5}}$ for $k=5, \ldots, 20$. Applying the inequality above gives

$$
A_{c}(1) \leq \sum_{k=0}^{4} 3^{k} \mathrm{e}^{-2^{k}}+16 \cdot 3^{5} \mathrm{e}^{-2^{5}}-\sum_{k=0}^{4} \sum_{j=0}^{20} 3^{k} \mathrm{e}^{-2^{k+1}} \frac{2^{k j}}{j !}+\frac{1}{21 !}\left(\frac{1}{2}\right)^{21} .
$$


Putting $A_{a}(1), A_{b}(1)$, and $A_{c}(1)$ together from (17), (18), and (19) shows that

$$
\begin{aligned}
A(1) \leq & \sum_{|k| \leq 20} \sum_{j=0}^{20} 3^{k} \mathrm{e}^{-2^{k+1}} 2^{k j} \frac{1}{j !} \mathrm{E}[M(j)]+\sum_{k=0}^{4} 3^{k} \mathrm{e}^{-2^{k}}-\sum_{k=0}^{4} \sum_{j=0}^{20} 3^{k} \mathrm{e}^{-2^{k+1}} \frac{2^{k j}}{j !} \\
& +\frac{1}{21 !}\left(\frac{1}{2}\right)^{21}+2\left(\frac{3}{\mathrm{e}^{2}}\right)^{21}+\frac{1}{2 \cdot 3^{20}}+16 \cdot 3^{5} \mathrm{e}^{-2^{5}} .
\end{aligned}
$$

An upper bound for $B(1)$. To bound $B(1)$, split it into two sums, then remove a small finite number of terms from each, and bound the tails. From (16),

$$
B(1):=\sum_{k=-\infty}^{\infty} 3^{k} \mathrm{e}^{-2^{k}}=\sum_{k=0}^{4} 3^{k} \mathrm{e}^{-2^{k}}+\sum_{k=1}^{12}\left(\frac{1}{3}\right)^{k} \mathrm{e}^{-2^{-k}}+\sum_{k=5}^{\infty} 3^{k} \mathrm{e}^{-2^{k}}+\sum_{k=13}^{\infty}\left(\frac{1}{3}\right)^{k} \mathrm{e}^{-2^{-k}} .
$$

Bounding $\mathrm{e}^{-2^{-k}}$ above by 1 in the last summation produces an upper bound of

$$
B(1) \leq \sum_{k=0}^{4} 3^{k} \mathrm{e}^{-2^{k}}+\sum_{k=1}^{12}\left(\frac{1}{3}\right)^{k} \mathrm{e}^{-2^{-k}}+3^{5} \sum_{k=5}^{\infty} 3^{k-5} \mathrm{e}^{-2^{k}}+\frac{1}{2 \cdot 3^{12}}
$$

Simple calculus shows that $2^{x} \geq 2^{5}-2(x-5)$ for $x \geq 5$. Thus,

$$
\mathrm{e}^{-2^{k}} \leq \mathrm{e}^{-2^{5}} \mathrm{e}^{-2(k-5)} \quad \text { for all } k \geq 5
$$

Using (21), we obtain

$$
B(1) \leq \sum_{k=0}^{4} 3^{k} \mathrm{e}^{-2^{k}}+\sum_{k=1}^{12}\left(\frac{1}{3}\right)^{k} \mathrm{e}^{-2^{-k}}+3^{5} \mathrm{e}^{-2^{5}} \sum_{k=5}^{\infty} 3^{k-5} \mathrm{e}^{-2(k-5)}+\frac{1}{2 \cdot 3^{12}} .
$$

Re-indexing the third sum to start at $k=0$ produces

$$
B(1) \leq \sum_{k=0}^{4} 3^{k} \mathrm{e}^{-2^{k}}+\sum_{k=1}^{12}\left(\frac{1}{3}\right)^{k} \mathrm{e}^{-2^{-k}}+3^{5} \mathrm{e}^{-2^{5}} \sum_{k=0}^{\infty}\left(\frac{3}{\mathrm{e}^{2}}\right)^{k}+\frac{1}{2 \cdot 3^{12}},
$$

which is bounded above by

$$
B(1) \leq \sum_{k=0}^{4} 3^{k} \mathrm{e}^{-2^{k}}+\sum_{k=1}^{12}\left(\frac{1}{3}\right)^{k} \mathrm{e}^{-2^{-k}}+2 \cdot 3^{5} \mathrm{e}^{-2^{5}}+\frac{1}{2 \cdot 3^{12}}
$$

which is the upper bound for $B(1)$.

A lower bound for $C(1)$ and an upper bound for $\tilde{\mathcal{L}}(1)$. To obtain a lower bound for $C(1)$, note that all the terms are positive, so dropping all the terms except where $-6 \leq k \leq 4$ gives a lower bound. From (16),

$$
C(1):=\frac{1}{6} \sum_{k=-\infty}^{\infty} 6^{k} \mathrm{e}^{-2^{k}}>\frac{1}{6} \sum_{k=0}^{4} 6^{k} \mathrm{e}^{-2^{k}}+\frac{1}{6} \sum_{k=1}^{6}\left(\frac{1}{6}\right)^{k} \mathrm{e}^{-2^{-k}}
$$


Using definition (16) and the bounds (20), (22), and (23), gives

$$
\begin{aligned}
\tilde{L}(1):= & A(1)+B(1)-C(1) \\
< & \sum_{|k| \leq 20} \sum_{j=0}^{20} 3^{k} \mathrm{e}^{-2^{k+1}} 2^{k j} \frac{1}{j !} \mathrm{E}[M(j)]+2 \sum_{k=0}^{4} 3^{k} \mathrm{e}^{-2^{k}}+\sum_{k=1}^{12}\left(\frac{1}{3}\right)^{k} \mathrm{e}^{-2^{-k}} \\
& -\sum_{k=0}^{4} \sum_{j=0}^{20} 3^{k} \mathrm{e}^{-2^{k+1}} \frac{2^{k j}}{j !}-\frac{1}{6} \sum_{k=0}^{4} 6^{k} \mathrm{e}^{-2^{k}}-\frac{1}{6} \sum_{k=1}^{6}\left(\frac{1}{6}\right)^{k} \mathrm{e}^{-2^{-k}} \\
& +\frac{1}{21 !}\left(\frac{1}{2}\right)^{21}+2\left(\frac{3}{\mathrm{e}^{2}}\right)^{21}+\frac{1}{2 \cdot 3^{20}}+16 \cdot 3^{5} \mathrm{e}^{-2^{5}}+2 \cdot 3^{5} \mathrm{e}^{-2^{5}}+\frac{1}{2 \cdot 3^{12}} \\
:= & \tilde{\mathcal{L}}^{\mathrm{u}}(1) .
\end{aligned}
$$

3.4.2. Analysis of $\tilde{\mathscr{L}}(3)$. Recall from (16) that $\tilde{\mathscr{L}}(3):=A(3)+B(3)-C(3)$. The idea is to obtain a lower bound, $\tilde{\mathscr{L}}_{1}(3)$, for $\tilde{\mathcal{L}}(3)$. This will be accomplished by finding lower bounds for $A(3)$ and $B(3)$, and combining these with an upper bound for $C(3)$.

A lower bound for A(3). Using definition (16) and conditioning on the value of the random number $\operatorname{Po}\left(2^{k}\right)$,

$$
\begin{aligned}
A(3) & :=3^{1 / D} \sum_{k=-\infty}^{\infty} 3^{k} \mathrm{e}^{-3 \cdot 2^{k}} \mathrm{E}\left[M\left(\operatorname{Po}\left(3 \cdot 2^{k}\right)\right)\right] \\
& =3^{1 / D} \sum_{k=-\infty}^{\infty} 3^{k} \mathrm{e}^{-3 \cdot 2^{k}} \sum_{j=0}^{\infty} \frac{\mathrm{e}^{-3 \cdot 2^{k}}\left(3 \cdot 2^{k}\right)^{j}}{j !} \mathrm{E}[M(j)] .
\end{aligned}
$$

Summing over only $|k| \leq 20$ and $0 \leq j \leq 20$ gives a lower bound of

$$
A(3)>3^{1 / D} \sum_{|k| \leq 20} \sum_{j=0}^{20} 3^{k} \mathrm{e}^{-6 \cdot 2^{k}} \frac{\left(3 \cdot 2^{k}\right)^{j}}{j !} \mathrm{E}[M(j)] .
$$

A lower bound for $B(3)$. Summing over only $-14 \leq k \leq 3$ in the definition of $B(3)$ given in (16) gives a lower bound of

$$
B(3)>3^{1 / D} \sum_{k=0}^{3} 3^{k} \mathrm{e}^{-3 \cdot 2^{k}}+3^{1 / D} \sum_{k=1}^{14}\left(\frac{1}{3}\right)^{k} \mathrm{e}^{-3 \cdot 2^{-k}} .
$$

An upper bound for $C(3)$ and a lower bound for $\tilde{\mathcal{L}}(3)$. By the definition of $\mathrm{C}(3)$ given in (16), splitting the sum into three parts produces

$$
C(3)=\frac{1}{6} 3^{1 / D+1} \sum_{k=-10}^{1} 6^{k} \mathrm{e}^{-3 \cdot 2^{k}}+\frac{1}{6} 3^{1 / D+1} \sum_{k=2}^{\infty} 6^{k} \mathrm{e}^{-3 \cdot 2^{k}}+\frac{1}{6} 3^{1 / D+1} \sum_{k=11}^{\infty}\left(\frac{1}{6}\right)^{k} \mathrm{e}^{-3 \cdot 2^{-k}} .
$$

Note that $2^{k} \geq 2 k$ when $k \geq 2$; thus,

$$
C(3) \leq 3^{1 / D+1} \sum_{k=-10}^{1} 6^{k-1} \mathrm{e}^{-3 \cdot 2^{k}}+3^{1 / D+1} \sum_{k=2}^{\infty} 6^{k-1} \mathrm{e}^{-6 k}+3^{1 / D+1} \sum_{k=11}^{\infty}\left(\frac{1}{6}\right)^{k+1} .
$$


Re-indexing the second and third summations gives

$$
\begin{aligned}
C(3) \leq & 3^{1 / D+1} \sum_{k=-10}^{1} 6^{k-1} \mathrm{e}^{-3 \cdot 2^{k}} \\
& +3^{1 / D+1}\left(\frac{6}{\mathrm{e}^{12}}+\frac{36}{\mathrm{e}^{24}}+\frac{6^{3}}{\mathrm{e}^{48}} \sum_{k=0}^{\infty}\left(\frac{6}{\mathrm{e}^{12}}\right)^{k}\right)+3^{1 / D+1}\left(\frac{1}{6}\right)^{12} \sum_{k=0}^{\infty}\left(\frac{1}{6}\right)^{k} .
\end{aligned}
$$

Thus,

$$
C(3)<3^{1 / D+1} \sum_{k=-10}^{1} 6^{k-1} \mathrm{e}^{-3 \cdot 2^{k}}+3^{1 / D+1}\left(\frac{6}{\mathrm{e}^{12}}+\frac{36}{\mathrm{e}^{24}}+\frac{217}{\mathrm{e}^{48}}\right)+3^{1 / D+1} \frac{1}{5 \cdot 6^{11}},
$$

since

$$
6^{3} \sum_{k=0}^{\infty}\left(\frac{6}{\mathrm{e}^{6}}\right)^{k}<217 \text { and } \sum_{k=0}^{\infty}\left(\frac{1}{6}\right)^{k}=\frac{6}{5} .
$$

Putting the bounds together for $A(3), B(3)$, and $C$ (3) from (25), (26), and (27) gives the desired lower bound:

$$
\begin{aligned}
\tilde{\mathcal{L}}(3)> & 3^{1 / D} \sum_{|k| \leq 20} \sum_{j=0}^{20} 3^{k} \mathrm{e}^{-6 \cdot 2^{k}} \frac{\left(3 \cdot 2^{k}\right)^{j}}{j !} \mathrm{E}[M(j)]+3^{1 / D} \sum_{k=0}^{3} 3^{k} \mathrm{e}^{-3 \cdot 2^{k}} \\
& +3^{1 / D} \sum_{k=1}^{14}\left(\frac{1}{3}\right)^{k} \mathrm{e}^{-3 \cdot 2^{-k}}-3^{1 / D+1} \sum_{k=-10}^{1} 6^{k-1} \mathrm{e}^{-3 \cdot 2^{k}} \\
& -3^{1 / D+1}\left(\frac{6}{\mathrm{e}^{12}}+\frac{36}{\mathrm{e}^{24}}+\frac{217}{\mathrm{e}^{48}}\right)-3^{1 / D+1} \frac{1}{5 \cdot 6^{11}} \\
:= & \tilde{\mathcal{L}}_{1}(3) .
\end{aligned}
$$

3.4.3. Evaluation of $\mathrm{E}[M(n)]$. In order to evaluate $\tilde{\mathcal{L}}^{\mathrm{u}}(1)$ and $\tilde{\mathcal{L}}_{1}(3)$, the values of $\mathrm{E}[M(n)]$ for $0 \leq n \leq 20$ must be computed. Recall that $M(n)$ is the random variable representing the distance from the origin to it nearest neighbor if there are exactly $n$ points uniformly distributed on $\mathcal{C}$. If $\mathcal{C}_{1}$ contains at least one point then the value of $M(n)$ is completely determined by the points in $\mathcal{C}_{1}$, and it is not affected by the points in $\mathcal{C}_{2}$. The only way that $M(n)$ will depend on the points in $\mathcal{C}_{2}$ is if there are no points in $\mathcal{C}_{1}$. We condition on the number of points which are located in $\mathcal{C}_{1}$. Since the probability of $k$ points in $\mathcal{C}_{1}$ is $\left(\begin{array}{l}n \\ k\end{array}\right) / 2^{n}$ and $\mathrm{E}\left[M\left(k|| \mathcal{C}_{1} \mid=k\right)\right]=\mathrm{E}[M(k)] / 3$, we see that

$$
\mathrm{E}[M(n)]=\frac{1}{2^{n}} \sum_{k=1}^{n}\left(\begin{array}{l}
n \\
k
\end{array}\right) \frac{1}{3} \mathrm{E}[M(k)]+\frac{1}{2^{n}}\left(\begin{array}{l}
n \\
0
\end{array}\right)\left(\frac{1}{3} \mathrm{E}[M(n)]+\frac{2}{3}\right),
$$

where the last term is obtained by conditioning on zero points in $\mathcal{C}_{1}$. Writing

$$
\mathrm{E}[M(n)]=\frac{1}{2^{n}} \sum_{=1}^{n-1}\left(\begin{array}{l}
n \\
k
\end{array}\right) \frac{1}{3} \mathrm{E}[M(k)]+\frac{1}{3 \cdot 2^{n-1}}+\frac{2}{3 \cdot 2^{n}} \mathrm{E}[M(n)],
$$


and solving for $\mathrm{E}[M(n)]$ yields

$$
\mathrm{E}[M(n)]=\frac{3 \cdot 2^{n-1}}{3 \cdot 2^{n-1}-1} \frac{1}{3 \cdot 2^{n}} \sum_{k=1}^{n-1}\left(\begin{array}{l}
n \\
k
\end{array}\right) \mathrm{E}[M(k)]+\frac{3 \cdot 2^{n-1}}{3 \cdot 2^{n-1}-1} \frac{1}{3 \cdot 2^{n-1}} .
$$

Thus, a recursive formula for $\mathrm{E}[M(n)]$ for $n \geq 2$ is given by

$$
\mathrm{E}[M(n)]=\frac{1}{3 \cdot 2^{n}-2} \sum_{k=1}^{n-1}\left(\begin{array}{l}
n \\
k
\end{array}\right) \mathrm{E}[M(k)]+\frac{2}{3 \cdot 2^{n}-2} .
$$

By symmetry, it is easily seen that $\mathrm{E}[M(1)]=\frac{1}{2}$. Note that $\mathrm{E}[M(n)]$ is only needed for $1 \leq n \leq 20$ (see (24) and (28)). To compute $\mathrm{E}[M(n)]$ for such values, use $\mathrm{E}[M(0)]=0$ and $\mathrm{E}[M(1)]=\frac{1}{2}$ along with recursion (29). These values are explicitly determined in Appendix A. $\tilde{\mathscr{L}}_{1}(3)$ :

By (24) and (28), together with the computed values for $\mathrm{E}[M(n)]$, we bound $\tilde{\mathcal{L}}^{\mathrm{u}}(1)$ and

$$
\tilde{\mathscr{L}}^{\mathrm{u}}(1) \leq 1.22789167 \quad \text { and } \quad \tilde{\mathcal{L}}_{1}(3) \geq 1.22790524 .
$$

Thus, putting everything together gives

$$
\tilde{\mathscr{L}}(1)<\tilde{\mathcal{L}}^{\mathrm{u}}(1) \leq 1.22789167<1.22790524 \leq \tilde{\mathcal{L}}_{1}(3)<\tilde{\mathscr{L}}(3),
$$

which shows that $\tilde{\mathcal{L}}$ is not constant, completing Step 3 .

\subsection{Proof of Step 4}

It is enough to show that $\tilde{\mathcal{L}}$ is continuous and strictly positive. Recall that $\tilde{\mathscr{L}}$ is $\log _{2}$ periodic by Step 2 . To show that $\tilde{\mathscr{L}}$ is continuous for all $\alpha>0$, it is enough to show, by periodicity, that $\tilde{\mathcal{L}}$ is continuous on $[1,2]$.

Since $\sum_{k=-\infty}^{\infty} 3^{k} \mathrm{e}^{-\alpha 2^{k}}$ and $\sum_{k=-\infty}^{\infty} 6^{k} \mathrm{e}^{-\alpha 2^{k}}$ are uniformly convergent for $\alpha \in(1-\varepsilon, 2+\varepsilon)$ with $0<\varepsilon<1$, it follows, by standard arguments (see, e.g. [3, p. 129]), that $B$ and $C$ are continuous functions on $\mathbb{R}^{+}$. To show that $A$ is a continuous function, again by standard arguments, it is enough to show that, for all $k \in \mathbb{Z}, \mathrm{E}\left[M\left(\operatorname{Po}\left(\alpha 2^{k}\right)\right)\right]$ is continuous for $\alpha \in$ $(1-\varepsilon, 2+\varepsilon)$.

Lemma 1. $\mathrm{E}\left[M\left(\operatorname{Po}\left(\alpha 2^{k}\right)\right)\right]$ is continuous for $\alpha \in(1-\varepsilon, 2+\varepsilon)$ with $0<\varepsilon<1$.

Fix $k \in \mathbb{Z}$. Since $0 \leq \mathrm{E}[M(j)] \leq 1$, it follows, for all $\alpha \in(1-\varepsilon, 2+\varepsilon)$ and all $k \in \mathbb{Z}$, that

$$
\sum_{j=0}^{\infty}\left|\frac{\left(\alpha 2^{k}\right)^{j}}{j !} \mathrm{E}[M(j)]\right| \leq \sum_{j=0}^{\infty} \frac{\left(3 \cdot 2^{k}\right)^{j}}{j !}=\mathrm{e}^{3 \cdot 2^{k}}<\infty .
$$

For fixed integers $j$ and $k$ and fixed $\varepsilon>0$, the mapping $\alpha \rightarrow\left(\alpha 2^{k}\right)^{j} \mathrm{E}[M(j)] / j$ ! is continuous on $(1-\varepsilon, 2+\varepsilon)$. So Weierstrass's M-test for uniform convergence proves that

$$
\mathrm{E}\left[M\left(\operatorname{Po}\left(\alpha 2^{k}\right)\right)\right]=\sum_{j=0}^{\infty} \frac{\left(\alpha 2^{k}\right)^{j}}{j !} \mathrm{E}[M(j)]
$$

is continuous for $\alpha \in(1-\varepsilon, 2+\varepsilon)$.

So $\tilde{\mathcal{L}}$ is continuous on $[1,2]$. Recall from (10) that $f(x)>0$ for all $x>0$ and from (15) that

$$
\tilde{\mathcal{L}}(\alpha)=(\alpha)^{1 / D-1}\left(\frac{3}{2}\right) \sum_{k=-\infty}^{\infty}\left(\frac{3}{2}\right)^{k} f\left(\alpha 2^{k}\right) .
$$

Thus, $\tilde{\mathscr{L}}$ is strictly positive and continuous for all $\alpha>0$, completing Step 4 . 


\subsection{Proof of Step 5}

To show Step 5, it suffices to show that, for all $\varepsilon>0$, there exists an $N(\varepsilon)$ such that, for all $n \geq N(\varepsilon)$,

$$
\left|\frac{\mathrm{E}[L(\operatorname{Po}(n))]}{n^{1 / D} \tilde{\mathcal{L}}(n)}-1\right|<\varepsilon .
$$

This is equivalent to showing that

$$
p_{n}(t):=\frac{\mathrm{E}\left[L\left(\operatorname{Po}\left(t 2^{n}\right)\right)\right]}{\left(t 2^{n}\right)^{1 / D} \tilde{\mathscr{L}}\left(t 2^{n}\right)} \rightarrow 1 \quad \text { as } n \rightarrow \infty,
$$

uniformly for $t \in[1,2]$.

Step 2 shows that $p_{n}(t)$ converges to 1 pointwise. By a similar argument to that in Step 4, it can be shown that $\mathrm{E}\left[L\left(\operatorname{Po}\left(t 2^{n}\right)\right)\right]$ is continuous for all $t \in[1,2]$. For each $n$, the mapping $t \rightarrow\left(t 2^{n}\right)^{1-1 / D} \tilde{\mathcal{L}}\left(t 2^{n}\right)$ is a continuous function and bounded away from 0 for $t \in[1,2]$. Thus, $p_{n}(t)$ is continuous on $[1,2]$.

By (1) we have

$$
\mathrm{E}\left[L\left(\operatorname{Po}\left(t 2^{n+1}\right)\right)\right]=\frac{2}{3} \mathrm{E}\left[L\left(\operatorname{Po}\left(t 2^{n}\right)\right)\right]+f\left(t 2^{n}\right) .
$$

Since $f>0$ and $\frac{2}{3}=2^{1-1 / D}$,

$$
\mathrm{E}\left[L\left(\operatorname{Po}\left(t 2^{n+1}\right)\right)\right]>2^{1-1 / D} \mathrm{E}\left[L\left(\operatorname{Po}\left(t 2^{n}\right)\right)\right] .
$$

Dividing both sides by $\left(t 2^{n+1}\right)^{1-1 / d} \tilde{\mathcal{L}}\left(t 2^{n+1}\right)$ and simplifying the right-hand side leads to

$$
p_{n+1}(t)=\frac{\mathrm{E}\left[L\left(\operatorname{Po}\left(t 2^{n+1}\right)\right)\right]}{\left(t 2^{n+1}\right)^{1-1 / d} \tilde{\mathcal{L}}\left(t 2^{n+1}\right)}>\frac{\mathrm{E}\left[L\left(\operatorname{Po}\left(t 2^{n}\right)\right)\right]}{\left(t 2^{n}\right)^{1-1 / d} \tilde{\mathcal{L}}\left(t 2^{n}\right)}=p_{n}(t) .
$$

We have a sequence of continuous functions on a compact set $X=[1,2]$, which is monotone increasing in $n$, that converges pointwise to 1 . So, by Dini's theorem we know that $p_{n}(t)$ converges uniformly, completing the proof of Step 5.

\section{Add-one cost}

In order to prove Theorem 2, an add-one bound is needed. The add-one result will show that there is a constant $c>0$ such that, for all $n \in \mathbb{N}$,

$$
|\mathrm{E}[L(n)]-\mathrm{E}[L(n+1)]| \leq c n^{-1 / D} .
$$

Similar add-one bounds exist in the context of $n$ uniform i.i.d. points on [0,1] (see [15, Statement 5.6]).

For every $y \in \mathbb{R}$ and $t \in \mathbb{R}^{+}$, let $B(y, t):=\{x \in \mathbb{R}:|x-y|<t\}$, i.e. the ball of radius $t$ centered at $y$. Let $\mu_{\mathcal{C}}(A)$ denote the Cantor measure of $A$ with respect to $\mathcal{C}$; see $[5$, p. 8] for more information. For example, $\mu_{\mathcal{C}}\left(\left[0, \frac{1}{3}\right]\right)=\frac{1}{2}$. To prove (30), it suffices to establish the following two inequalities:

$$
\mathrm{E}[L(n)] \leq \mathrm{E}[L(n+1)]+c n^{-1 / D} \text { and } \mathrm{E}[L(n+1)] \leq \mathrm{E}[L(n)]+c n^{-1 / D} .
$$

The add-one bound will rely on the following properties of the Cantor measure. Lemma 2 shows that the Cantor measure of any ball of radius $t$ is minimized if the ball is centered at the origin. This is what we would expect since the ball at the origin will only intersect the Cantor 
set to the right of the center. Lemma 2 also shows that the Cantor measure of any ball of radius $t$ is at most twice the Cantor measure of the ball of radius $t$ centered at the origin. The reasoning is that the most dense part of $\mathcal{C}$ occurs to the right of the origin, but twice the Cantor measure is needed since the left part of $B(0, t)$ has no Cantor measure.

Lemma 2. For all $y \in \mathcal{C}$ and $t \in[0,1]$,

$$
\mu_{\mathcal{C}}(B(0, t)) \leq \mu_{\mathcal{C}}(B(y, t)) \leq 2 \mu_{\mathcal{C}}(B(0, t)) .
$$

Proof. If $t=1$, the inequality holds trivially. Thus, we can assume that $t \neq 1$. We begin by proving the first inequality. Assume, by way of contradiction, that there exist a $y$ and $t$ such that

$$
\mu_{\mathcal{C}}(B(0, t))>\mu_{\mathcal{C}}(B(y, t)) .
$$

Choose $n \in \mathbb{N}$ such that $3^{-(n+1)} \leq t<3^{-n}$. By the symmetry of the Cantor set, there exists a $\tilde{y} \in \mathcal{C}$ such that $0 \leq \tilde{y} \leq 3^{-n}$ and $\mu_{\mathcal{C}}(B(y, t))=\mu_{\mathcal{C}}(B(\tilde{y}, t))$. If $0 \leq \tilde{y} \leq 3^{-(n+1)}$ then $B(0, t) \subseteq B(\tilde{y}, t)$, which implies that $\mu_{\mathcal{C}}(B(0, t)) \leq \mu_{\mathcal{C}}(B(\tilde{y}, t))$. This contradicts (31). Note that $\tilde{y} \notin\left(3^{-(n+1)}, 2 \cdot 3^{-(n+1)}\right)$ since $\tilde{y} \in \mathcal{C}$. Lastly, assume that $2 \cdot 3^{-(n+1)} \leq \tilde{y} \leq 3^{-n}$. By symmetry,

$$
\mu_{\mathcal{C}}(B(\tilde{y}, t))=\mu_{\mathcal{C}}\left(B\left(3^{-n}-\tilde{y}, t\right)\right),
$$

but $B(0, t) \subseteq B\left(3^{-n}-\tilde{y}, t\right)$. Thus,

$$
\mu_{\mathcal{C}}(B(0, t)) \leq \mu_{\mathcal{C}}\left(B\left(3^{-n}-\tilde{y}, t\right)\right)=\mu_{\mathcal{C}}(B(\tilde{y}, t))=\mu_{\mathcal{C}}(B(y, t)),
$$

which contradicts (31).

The second inequality is proved using Dobos's result [4] that the Cantor measure is subadditive at the origin, i.e. for all $a, b \in \mathbb{R}^{+}$,

$$
\mu_{\mathcal{C}}(B(0, a+b)) \leq \mu_{\mathcal{C}}(B(0, a))+\mu_{\mathcal{C}}(B(0, b)) .
$$

If $y>t$, applying subadditivity twice produces

$$
\begin{aligned}
\mu_{\mathcal{C}}(B(y, t)) & =\mu_{\mathcal{C}}(B(0, y+t))-\mu_{\mathcal{C}}(B(0, y-t)) \\
& \leq \mu_{\mathcal{C}}(B(0, y))+\mu_{\mathcal{C}}(B(0, t))-\mu_{\mathcal{C}}(B(0, y-t)) \\
& \leq \mu_{\mathcal{C}}(B(0, y-t))+\mu_{\mathcal{C}}(B(0, t))+\mu_{\mathcal{C}}(B(0, t))-\mu_{\mathcal{C}}(B(0, y-t)) \\
& =2 \mu_{\mathcal{C}}(B(0, t)) .
\end{aligned}
$$

If $y \leq t$ then $B(y, t) \subseteq B(0, y+t) \subseteq B(0,2 t)$. Thus, by subadditivity,

$$
\mu_{\mathcal{C}}(B(y, t)) \leq \mu_{\mathcal{C}}(B(0,2 t)) \leq 2 \mu_{\mathcal{C}}(B(0, t)),
$$

which completes the proof.

Finally, we require bounds for the expected distance between a point $y$ and its first and second nearest neighbors. Those bounds are needed because adding a point and going from a graph on $n$ points to a graph on $n+1$ points does not always imply an increase in the total edge length. The total edge length could decrease by the distance from $y$ to its nearest neighbor or second nearest neighbor. The appropriate bounds are found in the following lemma.

Let $F:=\left\{y_{1}, y_{2}, \ldots, y_{m}\right\}$ be a finite collection of points in $\mathcal{C}$. For any $y \in \mathcal{C}$ and $k \in\{1,2, \ldots, m\}$, let $d_{(k)}(y, F)$ be the distance from $y$ to its $k$ th nearest neighbor in the point set $F$. Adopt the notation that $d(y, F):=d_{(1)}(y, F)$. Now consider $\mathcal{X}_{n}:=\left\{X_{1}, X_{2}, \ldots X_{n}\right\}$ to be a random collection of $n$ vertices which are uniform i.i.d. on $\mathcal{C}$. 
Lemma 3. For all $y \in \mathcal{C}$,

(i) $\mathrm{E}\left[d\left(y, X_{n}\right)\right] \leq c n^{-1 / D}$, and

(ii) $\mathrm{E}\left[d_{(2)}\left(y, \chi_{n}\right)\right] \leq c n^{-1 / D}$.

Proof. Fix $y \in \mathcal{C}$. To prove part (i), consider

$$
\mathrm{E}\left[d\left(y, \chi_{n}\right)\right]=\int_{0}^{1} \mathrm{P}\left(d\left(y, X_{n}\right)>t\right) \mathrm{d} t .
$$

If $d\left(y, X_{n}\right)>t$ then $\mathcal{X}_{n} \cap B(y, t)=\varnothing$. By independence of the points in $\mathcal{X}_{n}$,

$$
\mathrm{P}\left(d\left(y, \mathcal{X}_{n}\right)>t\right)=\mathrm{P}\left(\mathcal{X}_{n} \cap B(y, t)=\varnothing\right)=\left(1-\mu_{\mathrm{C}}(B(y, t))\right)^{n} .
$$

However, by Lemma 2,

$$
\left(1-\mu_{\mathcal{C}}(B(y, t))\right)^{n} \leq\left(1-\mu_{\mathcal{C}}(B(0, t))\right)^{n} .
$$

Once again by independence of the points in $X_{n}$,

$$
\left(1-\mu_{\mathcal{C}}(B(0, t))\right)^{n}=\mathrm{P}\left(B(0, t) \cap \mathcal{X}_{n}=\varnothing\right)=\mathrm{P}\left(d\left(0, \mathcal{X}_{n}\right)>t\right) .
$$

So,

$$
\mathrm{P}\left(d\left(y, X_{n}\right)>t\right) \leq \mathrm{P}\left(d\left(0, X_{n}\right)>t\right) .
$$

Applying this to (32) gives

$$
\mathrm{E}\left[d\left(y, \chi_{n}\right)\right] \leq \int_{0}^{1} \mathrm{P}\left(d\left(0, X_{n}\right)>t\right) \mathrm{d} t=\mathrm{E}\left[d\left(0, \chi_{n}\right)\right] \leq c n^{-1 / D},
$$

where the last inequality follows from Statement (2.2) of Knopfmaker and Prodinger [8].

For the second nearest neighbor bound, part (ii), consider

$$
\mathrm{E}\left[d_{(2)}\left(y, x_{n}\right)\right]=\int_{0}^{1} \mathrm{P}\left(d_{(2)}\left(y, \chi_{n}\right)>t\right) \mathrm{d} t .
$$

If $d_{(2)}\left(y, X_{n}\right)>t$ then $X_{n} \cap B(y, t)$ contains at most one point. Thus, by independence of the points in $\mathcal{X}_{n}$,

$$
\begin{aligned}
\mathrm{P}\left(d_{(2)}\left(y, \mathcal{X}_{n}\right)>t\right) & =\mathrm{P}\left(\mathcal{X}_{n} \cap B(y, t)=\varnothing\right)+\mathrm{P}\left(\left|\mathcal{X}_{n} \cap B(y, t)\right|=1\right) \\
& =\left(1-\mu_{\mathcal{C}}(B(y, t))\right)^{n}+n\left(1-\mu_{\mathcal{C}}(B(y, t))\right)^{n-1} \mu_{\mathcal{C}}(B(y, t)) .
\end{aligned}
$$

However, by Lemma 2,

$$
n\left(1-\mu_{\mathcal{C}}(B(y, t))\right)^{n-1} \mu_{\mathcal{C}}(B(y, t)) \leq n\left(1-\mu_{\mathcal{C}}(B(0, t))\right)^{n-1} 2 \mu_{\mathcal{C}}(B(0, t)),
$$

and once again by independence of the points in $\mathcal{X}_{n}$,

$$
n\left(1-\mu_{\mathcal{C}}(B(0, t))\right)^{n-1} \mu_{\mathcal{C}}(B(0, t))=\mathrm{P}\left(\left|B(0, t) \cap \mathcal{X}_{n}\right|=1\right)=\mathrm{P}\left(d_{(2)}\left(0, X_{n}\right)>t\right) .
$$

Thus,

$$
\begin{aligned}
\mathrm{E}\left[d_{(2)}\left(y, \chi_{n}\right)\right] & \leq \int_{0}^{1}\left(1-\mu_{\mathcal{C}}(B(y, t))\right)^{n} \mathrm{~d} t+2 \int_{0}^{1} \mathrm{P}\left(d_{(2)}\left(0, \chi_{n}\right)>t\right) \mathrm{d} t \\
& =\mathrm{E}\left[d\left(y, \chi_{n}\right)\right]+2 \mathrm{E}\left[d_{(2)}\left(0, \chi_{n}\right)\right] .
\end{aligned}
$$

Knopfmaker and Prodinger [8] showed that $\mathrm{E}\left[d_{(2)}\left(0, \mathcal{X}_{n}\right)\right] \leq c n^{-1 / D}$ followed easily from [8, Statement (2.4)]. Combining this with (33), proves that (34) is bounded by $c n^{-1 / D}$. 
Now we prove the add-one bound.

Lemma 4. We have

$$
|\mathrm{E}[L(n)]-\mathrm{E}[L(n+k)]| \leq c k n^{-1 / D} .
$$

Proof. Let $\operatorname{deg}(v)$ denote the degree of the vertex $v$. Let $y \in \mathcal{C}$, and let $\mathcal{X}_{n+1}:=\mathcal{X}_{n} \cup\{y\}$. We first show that the inequality

$$
\mathrm{E}[L(n)] \leq \mathrm{E}[L(n+1)]+c n^{-1 / D}
$$

holds. The proof of (35) will consist of two cases depending on the relative location of $\mathcal{X}_{n}$ and $y$.

Case 1. Let $\Omega_{1}$ be the event $\operatorname{deg}(y)=2$ in the nearest-neighbor graph on $\mathcal{X}_{n+1}$ :

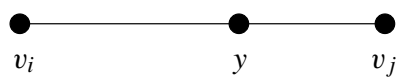

The nearest-neighbor graph on $\mathcal{X}_{n+1}$ is actually a graph for $\mathcal{X}_{n}$ where each vertex $v_{i} \in \mathcal{X}_{n}$ has a path to another vertex $v_{k} \in \mathcal{X}_{n}$. Thus, on $\Omega_{1}$, the length of the nearest-neighbor graph on $X_{n}$ is at most the length of the nearest-neighbor graph on $X_{n+1}$.

Case 2. Let $\Omega_{2}$ be the event $\operatorname{deg}(y)=1$ in the nearest-neighbor graph on $\mathcal{X}_{n+1}$ :

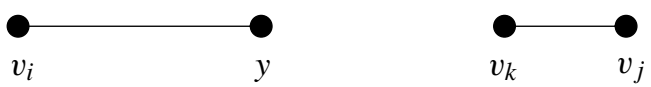

or

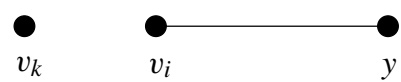

Adding the edge that connects $y$ to its second nearest neighbor, denoted by $v_{k}$, in $\mathcal{X}_{n}$ will produce a neighbors graph on $\mathcal{X}_{n}$ :

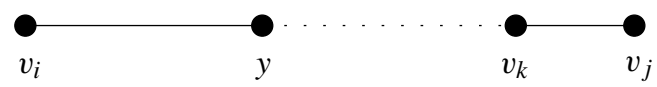

or

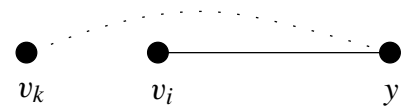

Thus, on $\Omega_{2}$, the length of the nearest-neighbor graph on $\mathcal{X}_{n+1}$ plus $d_{(2)}\left(y, \mathcal{X}_{n}\right)$ is greater than or equal to the length of the nearest-neighbor graph on $X_{n}$.

Conditioning on these two cases produces

$$
\begin{aligned}
\mathrm{E}[L(n)] & =\mathrm{E}\left[L(n) 1_{\Omega_{1}}\right]+\mathrm{E}\left[L(n) 1_{\Omega_{2}}\right] \\
& \leq \mathrm{E}\left[L(n+1) 1_{\Omega_{1}}\right]+\mathrm{E}\left[\left(L(n+1)+d_{(2)}\left(y, x_{n}\right)\right) 1_{\Omega_{2}}\right] \\
& \leq \mathrm{E}\left[\left(L(n+1)+d_{(2)}\left(y, \chi_{n}\right)\right) 1_{\Omega_{1}}\right]+\mathrm{E}\left[\left(L(n+1)+d_{(2)}\left(y, \chi_{n}\right)\right) 1_{\Omega_{2}}\right] .
\end{aligned}
$$

The desired inequality (35) follows by Lemma 3. Now we prove the reverse inequality

$$
\mathrm{E}[L(n+1)] \leq \mathrm{E}[L(n)]+c n^{-1 / D} .
$$


The nearest-neighbor graph on $\mathcal{X}_{n}$ along with the edge connecting $y$ to its nearest neighbor in $X_{n}$ produces a neighbors graph for $\mathcal{X}_{n+1}$; however, it may not be the nearest-neighbor graph. Thus,

$$
\mathrm{E}[L(n+1)] \leq \mathrm{E}[L(n)]+\mathrm{E}\left[d\left(y, \chi_{n}\right)\right]
$$

Applying Lemma 3 completes the proof of (36) and Lemma 4.

\section{Proof of Theorem 2}

Theorem 2 is proved by showing that the difference between the total edge length of the binomial and the Poisson nearest-neighbor graphs go to 0 as $n$ tends to $\infty$. The decay of a Poisson random variable will be enough to show that they have the same asymptotics.

Recall that

$$
n^{1 / D-1} \mathcal{L}(n) \mathrm{E}[L(\operatorname{Po}(n))] \rightarrow 1 \quad \text { as } n \rightarrow \infty
$$

In order to prove Theorem 2, it suffices to show the following proposition.

Proposition 1. We have

$$
n^{1 / D-1}[\mathrm{E}[L(n)]-\mathrm{E}[L(\operatorname{Po}(n))]] \rightarrow 0 \quad \text { as } n \rightarrow \infty .
$$

Proof. By conditioning on $\operatorname{Po}(n)$ we can decompose $|\mathrm{E}[L(n)]-\mathrm{E}[L(\operatorname{Po}(n))]|$ as follows:

$$
\begin{aligned}
|\mathrm{E}[L(n)]-\mathrm{E}[L(\operatorname{Po}(n))]|= & \left|\sum_{k=0}^{\infty}(\mathrm{E}[L(n)]-\mathrm{E}[L(k)]) \mathrm{P}(\mathrm{Po}(n)=k)\right| \\
\leq & \sum_{k=0}^{\infty}|\mathrm{E}[L(n)]-\mathrm{E}[L(k)]| \frac{\mathrm{e}^{-n} n^{k}}{k !} \\
= & \sum_{k=0}^{\lfloor n-6 \sqrt{n \log n}\rfloor}|\mathrm{E}[L(n)]-\mathrm{E}[L(k)]| \frac{\mathrm{e}^{-n} n^{k}}{k !} \\
& +\sum_{k=\lceil n-6 \sqrt{n \log n}\rceil}^{\lfloor n+6 \sqrt{n \log \rfloor}\rfloor}|\mathrm{E}[L(n)]-\mathrm{E}[L(k)]| \frac{\mathrm{e}^{-n} n^{k}}{k !} \\
& +\sum_{k=\lceil n+6 \sqrt{n \log n}\rceil}^{\infty}|\mathrm{E}[L(n)]-\mathrm{E}[L(k)]| \frac{\mathrm{e}^{-n} n^{k}}{k !} \\
:= & I+I I+I I I .
\end{aligned}
$$

Showing that

$$
n^{1 / D-1} I \rightarrow 0, \quad n^{1 / D-1} I I \rightarrow 0, \quad \text { and } \quad n^{1 / D-1} I I I \rightarrow 0, \quad \text { as } n \rightarrow \infty
$$

will complete the proof of Proposition 1. This is done in Sections 5.1, 5.2, and 5.3, respectively, with the help of the following Poisson probability bounds [1, p. 259]. 
Lemma 5. For all $m \in \mathbb{N}$,

(i)

$$
\mathrm{P}(\operatorname{Po}(n)=m) \leq \frac{1}{\sqrt{2 \pi m}} \exp \left(\frac{-(m-n)^{2}}{2(m+n)}\right)
$$

(ii)

$$
\mathrm{P}(\mathrm{Po}(n) \geq m) \leq \frac{m+1}{m+1-n} \mathrm{P}(\mathrm{Po}(n)=m) \quad \text { for } m+1>n,
$$

$$
\mathrm{P}(\mathrm{Po}(n) \leq m-1) \leq \frac{n}{n+1-m} \mathrm{P}(\operatorname{Po}(n)=m-1) \quad \text { for } m-1<n .
$$

\section{1. $n^{1 / D-1} I \rightarrow 0$ as $n \rightarrow \infty$}

Note that $L(k) \leq 1$ for all $k$ and, thus,

$$
n^{1 / D-1} I=2 n^{1 / D-1} \mathrm{P}(\operatorname{Po}(n) \leq\lfloor n-6 \sqrt{n \log n}\rfloor) .
$$

Letting $m:=\lfloor n-6 \sqrt{n \log n}\rfloor+1$ and applying Lemma 5(iii) gives

$$
n^{1 / D-1} I \leq 2 n^{1 / D-1} \frac{n}{n+1-(\lfloor n-6 \sqrt{n \log n}\rfloor+1)} \mathrm{P}(\operatorname{Po}(n)=\lfloor n-6 \sqrt{n \log n}\rfloor) .
$$

Applying Lemma 5(i) gives

$$
\begin{aligned}
n^{1 / D-1} I \leq & 2 n^{1 / D-1} \frac{n}{n-(\lfloor n-6 \sqrt{n \log n}\rfloor)} \frac{1}{\sqrt{2 \pi}} \\
& \times \frac{1}{\sqrt{\lfloor n-6 \sqrt{n \log n}\rfloor+1}} \exp \left(\frac{-(\lfloor n-6 \sqrt{n \log n}\rfloor+1-n)^{2}}{2(\lfloor n-6 \sqrt{n \log n}\rfloor+1+n)}\right) .
\end{aligned}
$$

Using the definition of the floor function produces

$$
n^{1 / D-1} I \leq c n^{1 / D-1} \frac{n}{n-(n-6 \sqrt{n \log n})} \frac{1}{\sqrt{n-6 \sqrt{n \log n}}} \exp \left(-\frac{(-6 \sqrt{n \log n})^{2}}{2(2 n+1-6 \sqrt{n \log n})}\right) .
$$

Simplifying and using crude bounds gives

$$
n^{1 / D-1} I \leq c n^{1 / D-1} n^{1 / 2}(1) \exp (-9 \log n)=c n^{1 / D-1 / 2} n^{-9}=c n^{1 / D-17 / 2},
$$

which will go to 0 as $n$ goes to $\infty$ since $1 / D<\frac{17}{2}$.

5.2. $n^{1 / D-1} I I \rightarrow 0$ as $n \rightarrow \infty$

Splitting the sum gives

$$
\begin{aligned}
n^{1 / D-1} I I= & n^{1 / D-1} \sum_{k=\lceil n-6 \sqrt{n \log n}\rceil}^{n}|\mathrm{E}[L(k+(n-k))]-\mathrm{E}[L(k)]| \frac{\mathrm{e}^{-n} n^{k}}{k !} \\
& +n^{1 / D-1} \sum_{k=n+1}^{\lfloor n+6 \sqrt{n \log n}\rfloor}|\mathrm{E}[L(n)]-\mathrm{E}[L(n+(k-n))]| \frac{\mathrm{e}^{-n} n^{k}}{k !} .
\end{aligned}
$$


Applying Lemma 4 to both sums gives

$$
\begin{aligned}
n^{1 / D-1} I I \leq & c n^{1 / D-1} \sum_{k=\lceil n-6 \sqrt{n \log n}\rceil}^{n}(n-k) k^{-1 / D} \frac{\mathrm{e}^{-n} n^{k}}{k !} \\
& +c n^{1 / D-1} \sum_{k=n+1}^{\lfloor n+6 \sqrt{n \log n}\rfloor}(k-n) n^{-1 / D} \frac{\mathrm{e}^{-n} n^{k}}{k !} .
\end{aligned}
$$

Using the upper and lower bounds for $k$ yields

$$
n^{1 / D-1} I I \leq c n^{1 / D-1}(\sqrt{n \log n})(n-6 \sqrt{n \log n})^{-1 / D}+c n^{1 / D-1}(\sqrt{n \log n}) n^{-1 / D},
$$

where $c$ is a constant that changes from line to line. Simplifying shows that

$$
n^{1 / D-1} I I \leq \frac{c \sqrt{\log n}}{\sqrt{n}}\left(1-\frac{6 \sqrt{\log n}}{\sqrt{n}}\right)^{-1 / D}+\frac{c \sqrt{\log n}}{\sqrt{n}}
$$

which goes to 0 as $n$ approaches $\infty$.

5.3. $n^{1 / D-1} I I I \rightarrow 0$ as $n \rightarrow \infty$

This term is handled similarly to term $I$. For all $k, L(k) \leq 1$; thus,

$$
n^{1 / D-1} I I I \leq 2 n^{1 / D-1} \mathrm{P}(\operatorname{Po}(n) \geq n+6 \sqrt{n \log n}) .
$$

Letting $m:=\lfloor n+6 \sqrt{n \log n}\rfloor$ and applying Lemma 5(i) and (ii) gives

$$
\begin{aligned}
n^{1 / D-1} I I I \leq & 2 n^{1 / D-1} \frac{\lfloor n+6 \sqrt{n \log n}\rfloor+1}{\lfloor n+6 \sqrt{n \log n}\rfloor+1-n} \frac{1}{\sqrt{2 \pi}} \frac{1}{\sqrt{\lfloor n+6 \sqrt{n \log n}\rfloor}} \\
& \times \exp \left(-\frac{(\lfloor n+6 \sqrt{n \log n}\rfloor-n)^{2}}{2(\lfloor n+6 \sqrt{n \log n}\rfloor+n)}\right) .
\end{aligned}
$$

Using bounds for the floor function yields

$$
n^{1 / D-1} I I I \leq c n^{1 / D-1} \frac{n+6 \sqrt{n \log n}+1}{6 \sqrt{n \log n}} \frac{1}{\sqrt{n+6 \sqrt{n \log n}-1}} \exp \left(-\frac{(6 \sqrt{n \log n}-1)^{2}}{2(2 n+6 \sqrt{n \log n})}\right)
$$

Simplifying and using crude bounds gives

$$
n^{1 / D-1} I I I \leq c n^{1 / D-1} \frac{n}{\sqrt{n}} \exp \left(-\frac{(5 \sqrt{n \log n})^{2}}{4 n}\right)=c n^{1 / D-27 / 4},
$$

which will go to 0 as $n$ goes to $\infty$ since $1 / D<\frac{27}{4}$. 
TABLE 1.

\begin{tabular}{rc}
\hline$n$ & $\mathrm{E}[M(n)]$ \\
\hline 0 & 0 \\
1 & $\frac{1}{2}$ \\
2 & $\frac{3}{10}$ \\
3 & $\frac{1}{5}$ \\
4 & $\frac{33}{230}$ \\
5 & $\frac{5}{46}$ \\
6 & $\frac{75}{874}$ \\
7 & $\frac{611}{8740}$ \\
8 & $\frac{97653}{1673710}$ \\
9 & $\frac{83057}{1673710}$ \\
10 & $\frac{22018179}{513828970}$ \\
11 & $\frac{9625216}{256914485}$ \\
12 & $\frac{20894487717}{631290272542}$ \\
13 & $\frac{93120706729}{3156451362710}$ \\
14 & $\frac{411117020063871}{15513958447719650}$ \\
15 & $\frac{297434062421057}{12411166758175720}$ \\
16 & $\frac{6650181371241300777}{305013731457236950790}$ \\
17 & $\frac{6082551300359191981}{305013731457236950790}$ \\
18 & $\frac{2198073713661546055399083}{119935974414957427604889850}$ \\
19 & 53388901948383223161199 \\
20 & 311228908583025195463286575 \\
198571427922359072046386438959 \\
\hline
\end{tabular}

\section{Appendix A. Evaluation of E[M(n)]}

Table 1 displays $\mathrm{E}[M(n)]$ for different values of $n$.

\section{Acknowledgements}

I would like to acknowledge the help of Joseph Yukich for proofreading and making suggestions on earlier drafts of this paper. He is an outstanding mentor and friend. I would also like to thank the anonymous referee for their thoughtful insight which produced a more intuitive exposition. 


\section{References}

[1] Barbour, A., Holst, L. And Janson, S. (1992). Poisson Approximation (Oxford Stud. Prob. 2). Oxford University Press.

[2] Baryshnikov, Y. AND YUKICH, J. E. (2005). Gaussian limits for random measures in geometric probability. Ann. Appl. Prob. 15, 213-253.

[3] Bromwich, T. J. (1942). An Introduction to the Theory of Infinite Series. Macmillan and Company.

[4] Dobos, J. (1996). The standard Cantor function is subadditive. Proc. Amer. Math. Soc. 124, 3425-3426.

[5] Durrett, R. (1996). Probability; Theory and Examples, 2nd edn. Duxbury Press, Belmont, CA.

[6] Gao, J. and Steele, J. M. (1994). General spacefilling curve heuristics and limit theory for the traveling salesman problem. J. Complexity 10, 230-245.

[7] Grabner, P. J. and Woess, W. (1997). Functional iterations and periodic oscillations for simple random walk on the Sierpiński graph. Stoch. Process. Appl. 69, 127-138.

[8] Knopfmacher, A. And Prodinger, H. (1996). Explicit and asymptotic formulae for the expected values of the order statistics of the Cantor distribution. Statist. Prob. Lett. 27, 189-194.

[9] Lalley, S. (1990). Traveling salesman with a self-similar itinerary. Prob. Eng. Inf. Sci. 4, 1-18.

[10] Penrose, M. (2000). Central limit theorems for $k$-nearest neighbour distances. Stoch. Process. Appl. 85, $295-320$.

[11] Penrose, M. D. And Yukich, J. E. (2002). Limit theory for random sequential packing and deposition. Ann. Appl. Prob. 12, 272-301.

[12] Penrose, M. D. And Yukich, J. E. (2003). Weak laws of large numbers in geometric probability. Ann. Appl. Prob. 13, 277-303.

[13] Platzman, L. K. and Bartholdi, J. J., III. (1989). Spacefilling curves and the planar traveling salesman problem. J. Assoc. Comput. Mach. 36, 719-737.

[14] Steele, J. M. (1997). Probability Theory and Combinatorial Optimization. Society for Industrial and Applied Mathematics, Philadelphia, PA.

[15] Yukich, J. E. (1998). Probability Theory of Classical Euclidean Optimization Problems (Lecture Notes Math. 1675). Springer, Berlin. 